\title{
Fixed point theorems in CAT(0) spaces with applications
}

Haishu Lu', Di Lan², Qingwen Hu ${ }^{3}$ and George Yuan ${ }^{4}$

This paper is dedicated to Professor Shih-Sen Chang for his 80 year's birthday.

"Correspondence:

dilan126@163.com

${ }^{2}$ School of Digital Media, Jiangnan

University, Wuxi, Jiangsu, 214122,

China

Full list of author information is

available at the end of the article

\begin{abstract}
In this paper, noncompact CAT(0) versions of the Fan-Browder fixed point theorem are established. As applications, we obtain new minimax inequalities, a saddle point theorem, a fixed point theorem for single-valued mappings, best approximation theorems, and existence theorems of $\varphi$-equilibrium points for multiobjective noncooperative games in the setting of noncompact CAT(0) spaces. These results generalize many well-known theorems in the literature.
\end{abstract}

MSC: $91 \mathrm{~A} 10 ; 47 \mathrm{H} 04 ; 47 \mathrm{H} 10 ; 54 \mathrm{H} 25$

Keywords: CAT(0) space; fixed point; minimax inequality; multiobjective game; equilibrium point

\section{Introduction}

Fixed point theorems for set-valued mappings play a vital role in various fields of pure and applied mathematics. In 1968, Browder [1] proved that every set-valued mapping with convex values and open fibers from a compact Hausdorff topological vector space to a convex space has a continuous selection. By using this selection theorem and the Brouwer fixed point theorem, Browder [1] obtained the famous Browder fixed point theorem which is equivalent to the Fan section theorem established by Fan [2] in 1961. For this reason, the Browder fixed point theorem is also called in the literature the Fan-Browder fixed point theorem. Since then, a body of generalizations and applications of the Fan-Browder fixed point theorem have been extensively investigated by many authors; see, for example, [312] and the references therein. In particular, Park [13] discussed some updated unified forms of KKM theorems under the framework of abstract convex spaces, which include hyperconvex spaces as special cases.

We recall that a $\mathrm{CAT}(0)$ space is a special metric space and it does not possess any linear structure. Many authors have made a lot of efforts to generalize the fixed point theory from Euclidean spaces to CAT(0) spaces. Recently, a number of authors pay attention to establish fixed point theorems in $\operatorname{CAT}(0)$ spaces. Kirk $[14,15]$ first studied the fixed point theory in $\operatorname{CAT}(0)$ spaces. Since then, many authors have developed the fixed point theory for single-valued and set-valued mappings in the setting of CAT(0) spaces. Dhompongsa et al. [16] proved that a nonexpansive mapping from a nonempty bounded closed convex subset of a CAT(0) space to the family of nonempty compact subsets of the CAT(0) space has a fixed point under suitable conditions. Shahzad [17] obtained fixed point theorems

@2014 Lu et al.: licensee Springer. This is an Open Access article distributed under the terms of the Creative Commons Attribution License (http://creativecommons.org/licenses/by/2.0), which permits unrestricted use, distribution, and reproduction in any medium, provided the original work is properly cited. 
for single-valued and set-valued mappings in $\mathrm{CAT}(0)$ spaces or $\mathbb{R}$-trees. By using a Ky Fan type minimax inequality in CAT(0) spaces, Shabanian and Vaezpour [18] proved fixed point theorems and best approximation theorems. More recently, Asadi [19] studied the existence problem of common fixed points for two mappings in CAT(0) spaces. Other results, we refer the reader to the literature of Kirk [20], Shahzad and Markin [21], Shahzad [17], and many others.

We know that both $\mathrm{CAT}(0)$ and hyperconvex spaces are two interesting classes of spaces. But a CAT(0) space may not be a hyperconvex, indeed a CAT(0) space is a hyperconvex space if and only if it is a complete $\mathbb{R}$-tree (see Kirk [22] and the references therein).

Inspired and motivated by the results mentioned above, in this paper, we first establish generalized $\mathrm{CAT}(0)$ versions of the Fan-Browder fixed point theorem. As applications, new minimax inequalities, a saddle point theorem, a fixed point theorem for singlevalued mappings, best approximation theorems, and existence theorems of $\varphi$-equilibrium points for multiobjective noncooperative games are obtained in the setting of noncompact CAT(0) spaces.

\section{Preliminaries}

Let $\mathbb{R}$ and $\mathbb{N}$ denote the set of all real numbers and the set of natural numbers, respectively. Let $X$ be a set. We will denote by $2^{X}$ the family of all subsets of $X$, by $\langle X\rangle$ the family of nonempty finite subsets of $X$. Let $A$ be a subset of a topological space $X$, we will denote the interior of $A$ in $X$ and the closure of $A$ in $X$ by $\operatorname{int}_{X} A$ and $\operatorname{cl}_{X} A$, respectively. Let $X$, $Y$ be two nonempty sets and $T: X \rightarrow 2^{Y}$ be a set-valued mapping. Then the set-valued mapping $T^{-1}: Y \rightarrow 2^{X}$ is defined by $T^{-1}(y)=\{x \in X: y \in T(x)\}$ for every $y \in Y$.

Now we introduce some notation and concepts related to CAT( 0$)$ spaces. For more details, the reader may consult $[16-19,21,23-29]$ and the references therein.

Let $(E, d)$ be a metric space. A geodesic which joints the pair of points $x_{1}, x_{2} \in E$ is a mapping $\gamma:[0, a] \subseteq \mathbb{R} \rightarrow E$ such that $\gamma(0)=x_{1}, \gamma(a)=x_{2}$, and $d\left(\gamma(t), \gamma\left(t^{\prime}\right)\right)=\left|t-t^{\prime}\right|$ for every $t, t^{\prime} \in[0, a]$. In particular, we have $a=d\left(x_{1}, x_{2}\right)$. The image $\gamma([0, a])$ of $\gamma$ is said to be a geodesic segment joining $x_{1}$ and $x_{2}$. If the segment $\gamma([0, a])$ is unique, then this geodesic segment is denoted by $\left[x_{1}, x_{2}\right]$. The metric space $(E, d)$ is said to be a geodesic space if, for every $x, y \in E$, there is a geodesic jointing $x$ and $y$, and $(E, d)$ is called to be uniquely geodesic if there is only one geodesic segment joining every pair of points $x, y \in E$.

Definition $2.1([18,29])$ Let $D$ be a subset of a geodesic space $(E, d)$. Then $D$ is said to be convex if every geodesic segment joining any two points in $D$ is contained in $D$.

A geodesic triangle $\Delta$ in a geodesic metric space $(E, d)$ consists of three points $x_{1}, x_{2}, x_{3} \in$ $E$ and a geodesic segment between each pair of $x_{1}, x_{2}, x_{3} \in E$. All these geodesic segments are called the edges of $\Delta$. A comparison triangle for the geodesic triangle $\Delta$ in $(E, d)$ is a triangle $\bar{\Delta}$ in the Euclidean plane $\mathbb{R}^{2}$ which consists of three vertices $\bar{x}_{1}, \bar{x}_{2}, \bar{x}_{3} \in \mathbb{R}^{2}$. The triangle $\bar{\Delta}$ has the same side lengths as $\Delta$. That is,

$$
d_{\mathbb{R}^{2}}\left(\bar{x}_{i}, \bar{x}_{j}\right)=d\left(x_{i}, x_{j}\right) \quad \text { for } i, j \in\{1,2,3\}
$$

We point out that such a comparison triangle always exists (see [23]). A geodesic space is said to be a $\operatorname{CAT}(0)$ space if the equality $d(x, y) \leq d_{\mathbb{R}^{2}}(\bar{x}, \bar{y})$ holds for every $x, y \in \Delta$ and every $\bar{x}, \bar{y} \in \bar{\Delta}$. Every $\operatorname{CAT}(0)$ space $(E, d)$ is uniquely geodesic (see [23]). 
Let $x, y_{1}, y_{2}$ be points in a $\operatorname{CAT}(0)$ space $(E, d)$ and $y_{0}$ be the midpoint of the segment $\left[y_{1}, y_{2}\right]$. Then the $\mathrm{CAT}(0)$ inequality implies the following inequality,

$$
d^{2}\left(x, y_{1}\right)+d^{2}\left(x, y_{2}\right) \geq 2 d^{2}\left(x, y_{0}\right)+\frac{1}{2} d^{2}\left(y_{1}, y_{2}\right)
$$

which is called the $(\mathrm{CN})$ inequality of Bruhat and Tits [30].

A subset of a $\operatorname{CAT}(0)$ space equipped with the induced metric, is a $\operatorname{CAT}(0)$ space if and only if it is convex (see [23]). Let $(E, d)$ be a $\mathrm{CAT}(0)$ space and $D \subseteq E$. Niculescu and Rovența [29] introduced the notion of a convex hull of $D$ as follows:

$$
\operatorname{co}(D)=\bigcup_{n=0}^{\infty} D_{n}
$$

where $D_{0}=D$ and for $n \geq 1$, the set $D_{n}$ consists of all points in $E$ which lie on geodesics which start and end in $D_{n-1}$.

Definition 2.2 ([29]) Let $D$ be a nonempty subset of a CAT(0) space $(E, d)$. A set-valued mapping $G: D \rightarrow 2^{E}$ is called to be a KKM mapping if

$$
\operatorname{co}(F) \subseteq \bigcup_{x \in F} G(x) \quad \text { for every } F \in\langle D\rangle
$$

Let $K$ be a nonempty subset of a topological space $X$. If every continuous mapping $\phi$ : $K \rightarrow K$ has a fixed point, then $K$ is said to have the fixed point property.

Definition 2.3 ([18]) A CAT $(0)$ space $(E, d)$ is said to have the convex hull finite property if the closed convex hull of every nonempty finite subset of $E$ has the fixed point property.

Lemma 2.1 ([29]) Let (E,d) be a complete CAT(0) space with the convex hull finite property and $X$ be a nonempty subset of E. Suppose that $H: X \rightarrow 2^{X}$ is a KKM mapping with closed values and $H(z)$ is compact for some $z \in X$. Then $\bigcap_{x \in X} H(x) \neq \emptyset$.

Lemma 2.2 Let $(E, d)$ be a complete metric space. Then $E$ is a geodesic space if and only if for every $x, y \in E$, there exists $m \in E$ such that $d(x, z)=d(z, y)=\frac{1}{2} d(x, y)$.

Proof The proof of sufficiency can be found in [23, p.4]. Therefore, it suffices to prove the necessity. By the definition of a geodesic space, for every $x, y \in E$, there exists a mapping $\gamma:[0, a] \subseteq \mathbb{R} \rightarrow E$ such that $\gamma(0)=x, \gamma(a)=y$, and $d\left(\gamma(t), \gamma\left(t^{\prime}\right)\right)=\left|t-t^{\prime}\right|$ for every $t, t^{\prime} \in$ $[0, a]$. Take $t_{0}=\frac{a}{2} \in[0, a]$ and $z=\gamma\left(t_{0}\right) \in E$. Then we have $d(x, z)=d\left(\gamma(0), \gamma\left(t_{0}\right)\right)=\frac{a}{2}$ and $d(z, y)=d\left(\gamma\left(t_{0}\right), \gamma(a)\right)=\frac{a}{2}$. Since $d(x, y)=d(\gamma(0), \gamma(a))=a$, it follows that $d(x, z)=d(z, y)=$ $\frac{1}{2} d(x, y)$. This completes the proof.

Lemma 2.3 ([23]) A geodesic space is a CAT(0) space if and only if it satisfies the $(C N)$ inequality.

Lemma 2.4 ([31]) Every locally compact $\mathrm{CAT}(0)$ space $(E, d)$ has the convex hull finite property. 
Lemma 2.5 ([25]) Let $(E, d)$ be a CAT(0) space and let $x, y \in E$. Then, for every $t \in[0,1]$, there exists a unique point $z \in[x, y]$ such that $d(x, z)=t d(x, y)$ and $d(y, z)=(1-t) d(x, y)$.

From now on, we will use the notation $(1-t) x \oplus t y$ for the unique point $z$ in Lemma 2.5.

Lemma 2.6 ([25]) Let $(E, d)$ be a CAT(0) space and let $x, y \in E$ such that $x \neq y$. Then $[x, y]=$ $\{(1-t) x \oplus t y: t \in[0,1]\}$.

\section{Fixed point theorems}

In this section, we will develop four new versions of fixed point theorems in noncompact $\mathrm{CAT}(0)$ spaces.

Theorem 3.1 Let $(E, d)$ be a complete CAT $(0)$ space with the convex hull finite property, $K$ be a nonempty compact subset of $E$, and $F, G: E \rightarrow 2^{E}$ be two set-valued mappings such that

(i) for every $y \in E, F(y) \subseteq G(y)$ and $G(y)$ is convex;

(ii) for every $x \in E, F^{-1}(x)$ is open in $E$;

(iii) for every $y \in K, F(y) \neq \emptyset$;

(iv) one of the following conditions holds:

(iv) ${ }_{1}$ for every $N \in\langle E\rangle$, there exists a nonempty compact convex subset $E_{N}$ of $E$ containing $N$ such that

$$
E_{N} \backslash K \subseteq \bigcup_{x \in E_{N}} \operatorname{int}_{E_{N}}\left(G^{-1}(x) \cap E_{N}\right) ;
$$

(iv) $)_{2}$ there exists a point $x_{0} \in E$ such that $\operatorname{cl}_{E}\left(E \backslash G^{-1}\left(x_{0}\right)\right) \subseteq K$.

Then there exists $\hat{y} \in E$ such that $\hat{y} \in G(\hat{y})$.

Proof We distinguish the following two cases (iv) $)_{1}$ and (iv) $)_{2}$ for the proof.

Case (iv) $)_{1}$. Suppose the contrary. Then, for every $y \in E$, we have $y \notin G(y)$. Define $\widetilde{G}, \widetilde{F}$ : $E \rightarrow 2^{E}$ by

$$
\begin{aligned}
& \widetilde{G}(x)=\operatorname{cl}_{E}\left(E \backslash G^{-1}(x)\right) \cap K, \quad x \in E, \\
& \widetilde{F}(x)=\left(E \backslash F^{-1}(x)\right) \cap K, \quad x \in E .
\end{aligned}
$$

We will prove that the family $\{\widetilde{G}(x): x \in E\}$ has the finite intersection property. Let $N \in\langle E\rangle$ be given. Then, by (iv) $)_{1}$, there exists a nonempty compact convex subset $E_{N}$ of $E$ containing $N$. Furthermore, we define two set-valued mappings $G^{\prime}, F^{\prime}: E_{N} \rightarrow 2^{E_{N}}$ by

$$
G^{\prime}(x)=\operatorname{cl}_{E_{N}}\left(E_{N} \backslash G^{-1}(x)\right) \text { and } \quad F^{\prime}(x)=E_{N} \backslash F^{-1}(x), \quad x \in E_{N} .
$$

By (i) and (ii), $G^{\prime}(x) \subseteq F^{\prime}(x)$ for every $x \in E_{N}$. Since $E_{N}$ is compact and every $G^{\prime}(x)$ is relatively closed in $E_{N}$, it follows that every $G^{\prime}(x)$ is compact. Now we show that the mapping $G^{*}: E_{N} \rightarrow 2^{E_{N}}$ defined by

$$
G^{*}(x)=E_{N} \backslash G^{-1}(x), \quad x \in E_{N},
$$


is a KKM mapping. Suppose the contrary. Then there exist $A \in\left\langle E_{N}\right\rangle$ and $y \in \operatorname{co}(A) \subseteq E_{N}$ such that

$$
y \notin \bigcup_{x \in A} G^{*}(x)=E_{N} \backslash \bigcap_{x \in A} G^{-1}(x) .
$$

Hence, we have $y \in \bigcap_{x \in A} G^{-1}(x)$ and $A \subseteq G(y)$. Therefore, we have $y \in \operatorname{co}(A) \subseteq G(y)$ by (i), which is a contradiction. Hence, $G^{*}$ is a KKM mapping and so is $G^{\prime}$. By Lemma 2.1 and (iv) $)_{1}$, we have

$$
\emptyset \neq \bigcap_{x \in E_{N}} G^{\prime}(x)=\bigcap_{x \in E_{N}} \mathrm{cl}_{E_{N}}\left(E_{N} \backslash G^{-1}(x)\right) \subseteq E_{N} \cap K .
$$

Taking $\hat{y} \in \bigcap_{x \in E_{N}} G^{\prime}(x)$ leads to

$$
\hat{y} \in \bigcap_{x \in E_{N}} G^{\prime}(x) \subseteq \bigcap_{x \in N}\left(G^{\prime}(x) \cap K\right) \subseteq \bigcap_{x \in N}\left(\operatorname{cl}_{E}\left(E \backslash G^{-1}(x)\right) \cap K\right)=\bigcap_{x \in N} \widetilde{G}(x),
$$

which implies that the family $\{\widetilde{G}(x): x \in E\}$ has the finite intersection property. By the compactness of $K$, we have $\bigcap_{x \in E} \widetilde{G}(x) \neq \emptyset$. Since $\widetilde{G}(x) \subseteq \widetilde{F}(x)$ for every $x \in E$, it follows that

$$
\begin{aligned}
\emptyset & \neq \bigcap_{x \in E} \widetilde{F}(x)=\bigcap_{x \in E}\left(E \backslash F^{-1}(x)\right) \cap K \\
& =\left(E \backslash \bigcup_{x \in E} F^{-1}(x)\right) \cap K \\
& =K \backslash \bigcup_{x \in E} F^{-1}(x) .
\end{aligned}
$$

By (iii), for every $y \in K, F(y) \neq \emptyset$ and so, $K \subseteq \bigcup_{x \in E} F^{-1}(x)$, which is a contradiction. Therefore, there exists $\hat{y} \in K$ such that $\hat{y} \in G(\hat{y})$. This completes the proof.

Case (iv) $)_{2}$. Suppose the contrary. Then, for every $y \in E, y \notin G(y)$. Now let us define two set-valued mappings $\widetilde{G}, \widetilde{F}: E \rightarrow 2^{E}$ by

$$
\begin{aligned}
& \widetilde{G}(x)=\operatorname{cl}_{E}\left(E \backslash G^{-1}(x)\right), \quad x \in E, \\
& \widetilde{F}(x)=E \backslash F^{-1}(x), \quad x \in E .
\end{aligned}
$$

By (i) and (ii), $\widetilde{G}(x) \subseteq \widetilde{F}(x)$ for every $x \in E$. We show that $\widetilde{G}$ is a KKM mapping. That is, for every $A \in\langle E\rangle, \operatorname{co}(A) \subseteq \bigcup_{x \in A} \widetilde{G}(x)$. Otherwise, there exist $A \in\langle E\rangle$ and a point $y \in \operatorname{co}(A)$ such that $y \notin \bigcup_{x \in A} \widetilde{G}(x)=E \backslash \bigcap_{x \in A} \operatorname{int}_{E} G^{-1}(x)$. It follows that $y \in \bigcap_{x \in A} G^{-1}(x)$. Therefore, $A \subseteq G(y)$. Since $G(y)$ is convex by (i), $y \in \operatorname{co}(A) \subseteq G(y)$, which is a contradiction. Hence, $\widetilde{G}$ is a KKM mapping. By the definition of $\widetilde{G}, \widetilde{G}(x)$ is closed in $E$ for every $x \in E$. By (iv) $)_{2}$, there exists a point $x_{0} \in E$ such that

$$
\widetilde{G}\left(x_{0}\right)=\operatorname{cl}_{E}\left(E \backslash G^{-1}\left(x_{0}\right)\right) \subseteq K,
$$

which implies that $\widetilde{G}\left(x_{0}\right)$ is compact. Then, by Lemma 2.1 , we get

$$
\emptyset \neq \bigcap_{x \in E} \widetilde{G}(x) \subseteq \widetilde{G}\left(x_{0}\right) \subseteq K .
$$


Therefore, we have

$$
\emptyset \neq K \cap\left(\bigcap_{x \in E} \widetilde{G}(x)\right) \subseteq K \cap\left(\bigcap_{x \in E} \widetilde{F}(x)\right) .
$$

Taking $y_{0} \in K \cap\left(\bigcap_{x \in E} \widetilde{F}(x)\right)$, we have $y_{0} \in K$ and $x \notin F\left(y_{0}\right)$ for every $x \in E$. Hence, we have $F\left(y_{0}\right)=\emptyset$, which contradicts (iii). Therefore, there exists $\hat{y} \in K$ such that $\hat{y} \in G(\hat{y})$. This completes the proof.

Remark 3.1 Theorem 3.1 can be regarded as a generalization of the Fan-Browder fixed point theorem on Euclidean spaces to CAT(0) spaces without any linear structure. Theorem 3.1 is different from Theorem 1 of Browder [1], Theorem 1 of Yannelis [3], and Theorem 2.4"' of Tan and Yuan [32], which are established in the setting of topological vector spaces.

Remark 3.2 If only (iv) ${ }_{1}$ of Theorem 3.1 holds, then the $E$ in Theorem 3.1 does not need to possess the convex hull finite property. In fact, from the first part of the proof of Theorem 3.1, we can see that for every $N \in\langle E\rangle, E_{N}$ is a nonempty compact convex subset of $E$ and thus, it is a compact CAT(0) space with the induced metric. Hence, by Lemma 2.4, $E_{N}$ has the convex hull finite property. The key approach to the first part of the proof of Theorem 3.1 is to define two set-valued mappings on each $E_{N}$ and then apply the KKM lemma on $E_{N}$. Therefore, the $E$ in Theorem 3.1 does not need to have the convex hull finite property.

Remark 3.3 If $F=G$, then (iv) 1 and (iv) $)_{2}$ of Theorem 3.1 can be replaced by the following equivalent conditions, respectively:

(iv) ${ }_{1}^{\prime}$ for every $N \in\langle E\rangle$, there exists a nonempty compact convex subset $E_{N}$ of $E$ containing $N$ such that $E_{N} \backslash K \subseteq \bigcup_{x \in E_{N}} F^{-1}(x)$;

(iv) ${ }_{2}^{\prime}$ there exists a point $x_{0} \in E$ such that $E \backslash F^{-1}\left(x_{0}\right) \subseteq K$.

Theorem 3.2 Let $(E, d)$ be a complete CAT(0) space with the convex hull finite property, $K$ be a nonempty compact subset of $E$, and $F, G: E \rightarrow 2^{E}$ be two set-valued mappings such that

(i) for every $y \in E, F(y) \subseteq G(y)$ and $G(y)$ is convex;

(ii) $K \subseteq \bigcup_{x \in E} \operatorname{int}_{E} F^{-1}(x)$;

(iii) one of the following conditions holds:

(iii) $)_{1}$ for every $N \in\langle E\rangle$, there exists a nonempty compact convex subset $E_{N}$ of $E$ containing $N$ such that

$$
E_{N} \backslash K \subseteq \bigcup_{x \in E_{N}} \operatorname{int}_{E_{N}}\left(G^{-1}(x) \cap E_{N}\right)
$$

(iii) 2 there exists a point $x_{0} \in E$ such that $\operatorname{cl}_{E}\left(E \backslash G^{-1}\left(x_{0}\right)\right) \subseteq K$.

Then there exists $\hat{y} \in E$ such that $\hat{y} \in G(\hat{y})$.

Proof Define $\widetilde{F}: E \rightarrow 2^{E}$ by $\widetilde{F}(y)=\left(\operatorname{int}_{E} F^{-1}\right)^{-1}(y)$ for every $y \in E$. By (i), we have $\widetilde{F}(y) \subseteq$ $F(y) \subseteq G(y)$ for every $y \in E$. By the definition of $\widetilde{F}$, we have $\widetilde{F}^{-1}(x)=\operatorname{int}_{E} F^{-1}(x)$ for every 
$x \in E$, which is open in $E$. By (ii) and by the definition of $\widetilde{F}$, we know that $\widetilde{F}(y) \neq \emptyset$ for every $y \in K$. Thus, all the hypotheses of Theorem 3.1 for $\widetilde{F}$ and $G$ are satisfied. Hence, by Theorem 3.1 for $\widetilde{F}$ and $G$, the conclusion of Theorem 3.2 holds.

Remark 3.4 We have shown that Theorem 3.1 implies Theorem 3.2. It is evident that Theorem 3.2 implies Theorem 3.1. Therefore, Theorem 3.1 is equivalent to Theorem 3.2.

By Theorem 3.1, we have the following maximal element theorem.

Theorem 3.3 Let $(E, d)$ be a complete $\mathrm{CAT}(0)$ space with the convex hull finite property, $K$ be a nonempty compact subset of $E$, and $F, G: E \rightarrow 2^{E}$ be two set-valued mappings such that

(i) for every $y \in E, F(y) \subseteq G(y)$ and $G(y)$ is convex;

(ii) for every $x \in E, F^{-1}(x)$ is open in $E$;

(iii) for every $y \in E, y \notin G(y)$;

(iv) one of the following conditions holds:

(iv) ${ }_{1}$ for every $N \in\langle E\rangle$, there exists a nonempty compact convex subset $E_{N}$ of $E$ containing $N$ such that

$$
E_{N} \backslash K \subseteq \bigcup_{x \in E_{N}} \operatorname{int}_{E_{N}}\left(G^{-1}(x) \cap E_{N}\right)
$$

(iv) $)_{2}$ there exists a point $x_{0} \in E$ such that $\operatorname{cl}_{E}\left(E \backslash G^{-1}\left(x_{0}\right)\right) \subseteq K$.

Then there exists $\hat{y} \in K$ such that $F(\hat{y})=\emptyset$.

Proof Suppose to the contrary that $F(y) \neq \emptyset$ for every $y \in K$. Then, by Theorem 3.1, there exists $\hat{y} \in E$ such that $\hat{y} \in G(\hat{y})$, which contradicts (iii) of Theorem 3.3. Therefore, the conclusion of Theorem 3.3 holds. This completes the proof.

Remark 3.5 Theorem 3.3 is equivalent to Theorem 3.1. We have shown that Theorem 3.1 implies Theorem 3.3. So, it suffices to show that Theorem 3.3 implies Theorem 3.1. Suppose not. Then, for every $y \in E, y \notin G(y)$. By Theorem 3.3, there exists $\hat{y} \in K$ such that $F(\hat{y})=\emptyset$, which contradicts (iii) of Theorem 3.1. Therefore, the conclusion of Theorem 3.1 holds.

Remark 3.6 Theorem 3.3 is established in the setting of noncompact CAT(0) spaces which include Hadamard manifolds as special cases (see $[23,33]$ and the references therein). Therefore, Theorem 3.3 generalizes Theorem 3.1 of Yang and $\mathrm{Pu}$ [34] from Hadamard manifolds to noncompact CAT $(0)$ spaces. We point out that the proof of Theorem 3.3 is different from that of Theorem 3.1 of Yang and $\mathrm{Pu}$ [34].

Let $I$ be a finite index set and $\left\{\left(E_{i}, d_{i}\right)\right\}_{i \in I}$ be a family of metric spaces, where $d_{i}$ is the metric of $E_{i}$ for every $i \in I$. Let $(E, d)$ be the product space $\prod_{i \in I}\left(E_{i}, d_{i}\right)$, where $d$ is the metric of $E$. For every $i \in I$, every $x_{i} \in E_{i}$, and every $r>0$, let $U_{i}^{d_{i}}\left(x_{i}, r\right) \subseteq E_{i}$ denote the open ball centered at $x_{i}$ with radius $r$. For every $x \in E$ and every $r>0$, let $U^{d}(x, r) \subseteq E$ denote the open ball centered at $x$ with radius $r$. 
By Theorem 3.1, we have the following collectively fixed point theorem in noncompact CAT(0) spaces.

Theorem 3.4 Let $\left\{\left(E_{i}, d_{i}\right)\right\}_{i \in I}$ be a family of complete locally compact CAT $(0)$ spaces, where $I$ is a finite index set. Let $K$ be a nonempty compact subset of $E=\prod_{i \in I} E_{i}$. For every $i \in I$, let $F_{i}, G_{i}: E \rightarrow 2^{E_{i}}$ be two set-valued mappings such that

(i) for every $i \in I$ and every $y \in E, F_{i}(y) \subseteq G_{i}(y)$ and $G_{i}(y)$ is convex;

(ii) for every $i \in I$ and every $x_{i} \in E_{i}, F_{i}^{-1}\left(x_{i}\right)$ is open in $E$;

(iii) for every $i \in I$ and every $y \in K, F_{i}(y) \neq \emptyset$;

(iv) one of the following conditions holds:

(iv) ${ }_{1}$ for every $i \in I$ and every $N_{i} \in\left\langle E_{i}\right\rangle$, there exists a nonempty compact convex subset $E_{N_{i}}$ of $E_{i}$ containing $N_{i}$ such that, for every $y=\left(y_{i}\right)_{i \in I} \in E_{N} \backslash K$, there exist $r(y)>0$ and $\bar{x}(y)=\left(\bar{x}_{i}(y)\right)_{i \in I} \in E_{N}$ such that

$$
\prod_{i \in I} U_{i}^{d_{i}}\left(y_{i}, r(y)\right) \cap E_{N} \subseteq \bigcap_{i \in I} G_{i}^{-1}\left(\bar{x}_{i}(y)\right) \cap E_{N},
$$

where $E_{N}=\prod_{i \in I} E_{N_{i}}$;

(iv) $)_{2}$ there exists a point $x_{0}=\left(x_{0 i}\right)_{i \in I} \in E$ such that $\operatorname{cl}_{E}\left(E \backslash \bigcap_{i \in I} G_{i}^{-1}\left(x_{0 i}\right)\right) \subseteq K$.

Then there exists $\hat{y} \in E$ such that $\hat{y}_{i} \in G_{i}(\hat{y})$ for every $i \in I$.

Proof Let $I=\{1,2, \ldots, n\}$. Define $d: E \times E \rightarrow \mathbb{R}$ by

$$
d(x, y)=\sqrt{\sum_{i=1}^{n} d_{i}^{2}\left(x_{i}, y_{i}\right)}, \quad x=\left(x_{1}, x_{2}, \ldots, x_{n}\right) \in E, y=\left(y_{1}, y_{2}, \ldots, y_{n}\right) \in E .
$$

We prove Theorem 3.4 in the following four steps.

Step 1 . Show that $(E, d)$ is a metric space.

In fact, it suffices to check the triangle inequality; that is, for every $x=\left(x_{1}, x_{2}, \ldots, x_{n}\right), y=$ $\left(y_{1}, y_{2}, \ldots, y_{n}\right), z=\left(z_{1}, z_{2}, \ldots, z_{n}\right) \in E$, we have $d(x, y) \leq d(x, z)+d(z, y)$. In order to prove it, we have to show that

$$
\sum_{i=1}^{n} d_{i}^{2}\left(x_{i}, y_{i}\right) \leq d_{i}^{2}\left(x_{i}, z_{i}\right)+d_{i}^{2}\left(z_{i}, y_{i}\right)+2 \sqrt{\sum_{i=1}^{n} d_{i}^{2}\left(x_{i}, z_{i}\right)} \sqrt{\sum_{i=1}^{n} d_{i}^{2}\left(z_{i}, y_{i}\right)}
$$

By the Cauchy-Schwarz inequality, we have

$$
\sum_{i=1}^{n} d_{i}\left(x_{i}, z_{i}\right) d_{i}\left(z_{i}, y_{i}\right) \leq \sqrt{\sum_{i=1}^{n} d_{i}^{2}\left(x_{i}, z_{i}\right)} \sqrt{\sum_{i=1}^{n} d_{i}^{2}\left(z_{i}, y_{i}\right)} .
$$

Thus, we get

$$
\begin{aligned}
\sum_{i=1}^{n}\left(d_{i}\left(x_{i}, z_{i}\right)+d_{i}\left(z_{i}, y_{i}\right)\right)^{2} & =\sum_{i=1}^{n} d_{i}^{2}\left(x_{i}, z_{i}\right)+\sum_{i=1}^{n} d_{i}^{2}\left(z_{i}, y_{i}\right)+2 \sum_{i=1}^{n} d_{i}\left(x_{i}, z_{i}\right) d_{i}\left(z_{i}, y_{i}\right) \\
& \leq \sum_{i=1}^{n} d_{i}^{2}\left(x_{i}, z_{i}\right)+\sum_{i=1}^{n} d_{i}^{2}\left(z_{i}, y_{i}\right)
\end{aligned}
$$




$$
\begin{aligned}
& +2 \sqrt{\sum_{i=1}^{n} d_{i}^{2}\left(x_{i}, z_{i}\right)} \sqrt{\sum_{i=1}^{n} d_{i}^{2}\left(z_{i}, y_{i}\right)} \\
& =(d(x, z)+d(z, y))^{2} .
\end{aligned}
$$

Since $d_{i}\left(x_{i}, y_{i}\right) \leq d_{i}\left(x_{i}, z_{i}\right)+d_{i}\left(z_{i}, y_{i}\right)$ for every $i \in\{1,2, \ldots, n\}$, it follows from the above inequality that $d(x, y) \leq d(x, z)+d(z, y)$.

Step 2 . Show that $(E, d)$ is a complete locally compact space.

Firstly, we show that the topology $\tau_{d}$ associated with the metric $d$ is the product topology on $E$. In fact, for every $x=\left(x_{1}, x_{2}, \ldots, x_{n}\right), y=\left(y_{1}, y_{2}, \ldots, y_{n}\right) \in E$, let $\delta(x, y)=\max \left\{d_{i}\left(x_{i}, y_{i}\right)\right.$ : $i=1,2, \ldots, n\}$. Then $\delta$ is a metric of $E$ and we have

$$
\delta(x, y) \leq d(x, y) \leq \sqrt{n} \delta(x, y), \quad(x, y) \in E \times E
$$

Therefore, the metric $\delta$ is equivalent to $d$ and hence, $\tau_{\delta}=\tau_{d}$. For every $r>0$ and every $x=$ $\left(x_{1}, x_{2}, \ldots, x_{n}\right) \in E$, we have $U^{\delta}(x, r)=\prod_{i=1}^{n} U_{i}^{d_{i}}\left(x_{i}, r\right)$. In fact, $y \in U^{\delta}(x, r) \Leftrightarrow \max \left\{d_{i}\left(x_{i}, y_{i}\right)\right.$ : $1 \leq i \leq n\}<r \Leftrightarrow d_{i}\left(x_{i}, y_{i}\right)<r$ for every $i \in\{1,2, \ldots, n\}$. So, $y \in U^{\delta}(x, r) \Leftrightarrow y_{i} \in U_{i}^{d_{i}}\left(x_{i}, r\right)$ for every $i \in\{1,2, \ldots, n\} \Leftrightarrow y \in \prod_{i=1}^{n} U_{i}^{d_{i}}\left(x_{i}, r\right)$. We can see that the collection $\left\{U^{\delta}(x, r): x \in\right.$ $E$ and $r>0\}$ forms a base for $\tau_{\delta}$ and the collection $\left\{\prod_{i=1}^{n} U_{i}^{d_{i}}\left(x_{i}, r\right): x_{i} \in E_{i}\right.$ and $\left.r>0\right\}$ forms a base for the product topology on $E$. Hence, $\left\{U^{\delta}(x, r): x \in E\right.$ and $\left.r>0\right\}$ also forms a base for the product topology on $E$. Therefore, the topology $\tau_{d}$ associated to the metric $d$ is the product topology on $E$.

Secondly, we prove that $E$ is a complete space. In fact, let $\left\{x^{(k)}\right\}_{k \geq 1}$ be a Cauchy sequence with points $x^{(k)}=\left(x_{i}^{(k)}\right)_{i \in I} \in E$. Thus, for every $\varepsilon>0$, there is a positive integer $n_{0}$ such that for all positive integers $k \geq n_{0}$ and $m \geq n_{0}, d\left(x^{k}, x^{m}\right)<\varepsilon$. Hence, for every $i \in\{1,2, \ldots, n\}$, $d_{i}\left(x_{i}^{(k)}, x_{i}^{(m)}\right) \leq d\left(x^{k}, x^{m}\right)<\varepsilon$ whenever $k \geq n_{0}$ and $m \geq n_{0}$, which implies that, for every $i \in\{1,2, \ldots, n\},\left\{x_{i}^{(k)}\right\}$ is a Cauchy sequence in $E_{i}$. Since every $E_{i}$ is a complete metric space, it follows that $\lim _{k \rightarrow \infty} x_{i}^{(k)}=x_{i}$ for every $i \in\{1,2, \ldots, n\}$. Let $\varepsilon>0$ be arbitrarily given. For every $i \in\{1,2, \ldots, n\}$, there exists a positive integer $k(i)$ such that

$$
d_{i}\left(x_{i}^{(k)}, x_{i}\right)<\frac{\varepsilon}{\sqrt{n}} \quad \text { for all } k \geq k(i)
$$

Consequently, we have

$$
d\left(x^{(k)}, x\right)=\sqrt{\sum_{i=1}^{n} d_{i}^{2}\left(x_{i}^{(k)}, x_{i}\right)}<\varepsilon \quad \text { for all } k \geq k^{\prime}=\max \{k(1), k(2), \ldots, k(n)\}
$$

Thus, $\lim _{k \rightarrow \infty} x^{(k)}=x=\left(x_{i}\right)_{i \in I} \in E$, which implies that $E$ is a complete metric space.

Finally, we show that $E$ is a locally compact space. Let $x=\left(x_{i}\right)_{i \in I} \in E$ be an arbitrary point. Since every $E_{i}$ is a locally compact space, it follows that, for every $i \in\{1,2, \ldots, n\}$, there exists $r_{i}>0$ such that $\mathrm{cl}_{E_{i}} U_{i}^{d_{i}}\left(x_{i}, r_{i}\right)$ is compact. Let $r=\min \left\{r_{1}, r_{2}, \ldots, r_{n}\right\}$. Then we have $U^{d}(x, r) \subseteq \prod_{i=1}^{n} U_{i}^{d_{i}}\left(x_{i}, r\right) \subseteq \prod_{i \in I} U_{i}^{d_{i}}\left(x_{i}, r_{i}\right)$, which implies that

$$
\operatorname{cl}_{E} U^{d}(x, r) \subseteq \operatorname{cl}_{E}\left(\prod_{i=1}^{n} U_{i}^{d_{i}}\left(x_{i}, r\right)\right) \subseteq \prod_{i=1}^{n} \mathrm{cl}_{E_{i}} U_{i}^{d_{i}}\left(x_{i}, r_{i}\right)
$$


Since $\prod_{i=1}^{n} \operatorname{cl}_{E_{i}} U_{i}^{d_{i}}\left(x_{i}, r_{i}\right)$ is compact and $\mathrm{cl}_{E} U^{d}(x, r)$ is closed, it follows that $\mathrm{cl}_{E} U^{d}(x, r)$ is compact. Hence, $E$ is locally compact.

Step 3. Show that $(E, d)$ is a $\operatorname{CAT}(0)$ space.

For every $x=\left(x_{1}, x_{2}, \ldots, x_{n}\right), y=\left(y_{1}, y_{2}, \ldots, y_{n}\right) \in E$. Since every $E_{i}$ is a complete CAT( 0$)$ space and thus, it is a geodesic space, it follows from Lemma 2.2 that there exists $z_{i} \in E_{i}$ such that $d_{i}\left(x_{i}, z_{i}\right)=d_{i}\left(z_{i}, y_{i}\right)=\frac{1}{2} d_{i}\left(x_{i}, y_{i}\right)$ for every $i \in\{1,2, \ldots, n\}$. Let $z=\left(z_{i}\right)_{i \in I} \in E$. Then we have

$$
d(x, z)=\sqrt{\sum_{i=1}^{n} d_{i}^{2}\left(x_{i}, z_{i}\right)}=\sqrt{\sum_{i=1}^{n} d_{i}^{2}\left(z_{i}, y_{i}\right)}=d(z, y)=\frac{1}{2} d(x, y) .
$$

Hence, by Lemma 2.2 again, $E$ is a geodesic space. Now we claim that $E$ satisfies the $(\mathrm{CN})$ inequality. In fact, let $x=\left(x_{1}, x_{2}, \ldots, x_{n}\right), y=\left(y_{1}, y_{2}, \ldots, y_{n}\right), z=\left(z_{1}, z_{2}, \ldots, z_{n}\right) \in E$ and $p=\left(p_{1}, p_{2}, \ldots, p_{n}\right) \in E$ with $d(y, p)=d(p, z)=\frac{1}{2} d(y, z)$. We show that $d_{i}\left(y_{i}, p_{i}\right)=d_{i}\left(p_{i}, z_{i}\right)=$ $\frac{1}{2} d_{i}\left(y_{i}, z_{i}\right)$ for every $i \in\{1,2, \ldots, n\}$. Let $\alpha$ and $\beta$ be two numbers satisfying $\alpha+\beta \geq 1$. Then $\alpha^{2}+\beta^{2} \geq \frac{1}{2}(\alpha+\beta)^{2} \geq \frac{1}{2}$ with equality if and only if $\alpha=\beta=\frac{1}{2}$. By this fact and by the triangle inequality, we get

$$
\left(\frac{d_{i}\left(y_{i}, p_{i}\right)}{d_{i}\left(y_{i}, z_{i}\right)}\right)^{2}+\left(\frac{d_{i}\left(p_{i}, z_{i}\right)}{d_{i}\left(y_{i}, z_{i}\right)}\right)^{2} \geq \frac{1}{2}, \quad i \in\{1,2, \ldots, n\}
$$

We can see that the left of the above inequality equals $\frac{1}{2}$ if and only if $\frac{1}{2} d_{i}\left(y_{i}, z_{i}\right)=d_{i}\left(y_{i}, p_{i}\right)=$ $d_{i}\left(p_{i}, z_{i}\right)$ for every $i \in\{1,2, \ldots, n\}$. Adding these inequalities leads to

$$
\frac{1}{2} \sum_{i=1}^{n} d_{i}^{2}\left(y_{i}, z_{i}\right) \leq \sum_{i=1}^{n} d_{i}^{2}\left(y_{i}, p_{i}\right)+\sum_{i=1}^{n} d_{i}^{2}\left(p_{i}, z_{i}\right)
$$

that is, $\frac{1}{2} d^{2}(y, z) \leq d^{2}(y, p)+d^{2}(p, z)$. Since $d(y, p)=d(p, z)=\frac{1}{2} d(y, z)$, it follows from the above inequality that $d_{i}\left(y_{i}, p_{i}\right)=d_{i}\left(p_{i}, z_{i}\right)=\frac{1}{2} d_{i}\left(y_{i}, z_{i}\right)$ for every $i \in\{1,2, \ldots, n\}$. Since every $E_{i}$ is a $\mathrm{CAT}(0)$ space, by Lemma 2.3 , we have the following $(\mathrm{CN})$ inequality:

$$
d_{i}^{2}\left(x_{i}, y_{i}\right)+d_{i}^{2}\left(x_{i}, z_{i}\right) \geq 2 d_{i}^{2}\left(x_{i}, p_{i}\right)+\frac{1}{2} d_{i}^{2}\left(y_{i}, z_{i}\right), \quad i \in\{1,2, \ldots, n\}
$$

Adding these inequalities, we get

$$
d^{2}(x, y)+d^{2}(x, z) \geq 2 d^{2}(x, p)+\frac{1}{2} d^{2}(y, z)
$$

which implies that $E$ satisfies the $(\mathrm{CN})$ inequality. By Lemma 2.3 again, we know that $E$ is a CAT(0) space.

Step 4. Prove that there exists $\hat{y} \in E$ such that $\hat{y}_{i} \in G_{i}(\hat{y})$ for every $i \in I$.

By the above steps, we know that $E$ is a complete locally compact CAT(0) space. Thus, by Lemma 2.4, $E$ has the convex hull finite property. Now we define two set-valued mappings $F, G: E \rightarrow 2^{E}$ by

$$
F(y)=\prod_{i \in I} F_{i}(y) \quad \text { and } \quad G(y)=\prod_{i \in I} G_{i}(y), \quad y \in E .
$$


By (i), $F(y) \subseteq G(y)$ for every $y \in E$. For every $x \in E$, we have

$$
\begin{aligned}
F^{-1}(x) & =\{y \in E: x \in F(y)\} \\
& =\left\{y \in E: x \in \prod_{i \in I} F_{i}(y)\right\} \\
& =\left\{y \in E: x_{i} \in F_{i}(y), \forall i \in I\right\} \\
& =\left\{y \in E: y \in F_{i}^{-1}\left(x_{i}\right), \forall i \in I\right\} \\
& =\left\{y \in E: y \in \bigcap_{i \in I} F_{i}^{-1}\left(x_{i}\right)\right\} \\
& =\bigcap_{i \in I} F_{i}^{-1}\left(x_{i}\right) .
\end{aligned}
$$

Then, by (ii) and by the fact that $I$ is a finite index set, we know that $F^{-1}(x)$ is open in $E$ for every $x \in E$. By (iii), for every $y \in K, F(y) \neq \emptyset$. Suppose that (iv) ${ }_{1}$ holds. Then, for every $N \in\langle E\rangle$ and every $N_{i} \in\left\langle E_{i}\right\rangle$, there exists a nonempty compact convex subset $E_{N_{i}}$ of $E_{i}$ containing $N_{i}$ and so, $E_{N_{i}}$ is naturally a compact $\mathrm{CAT}(0)$ space with the induced metric. By using the same method as in Step 3, we can prove that $E_{N}=\prod_{i \in I} E_{N_{i}} \ni N$ is a nonempty compact CAT( 0$)$ space and hence, it is naturally a nonempty compact convex subset of $E$. For every $y=\left(y_{1}, y_{2}, \ldots, y_{n}\right) \in E$ and every $r>0$, we can see that $U^{d}(y, r) \subseteq \prod_{i \in I} U_{i}^{d_{i}}\left(y_{i}, r\right)$. Therefore, by this fact and by (iv) ${ }_{1}$, for every $y=\left(y_{i}\right)_{i \in I} \in E_{N} \backslash K$, there exist $r(y)>0$ and $\bar{x}(y)=\left(\bar{x}_{i}(y)\right)_{i \in I} \in E_{N}$ such that

$$
\begin{aligned}
U^{d}(y, r(y)) \cap E_{N} & \subseteq \prod_{i \in I} U_{i}^{d_{i}}\left(y_{i}, r(y)\right) \cap E_{N} \\
& \subseteq \bigcap_{i \in I} G_{i}^{-1}\left(\bar{x}_{i}(y)\right) \cap E_{N} \\
& =G^{-1}(\bar{x}(y)) \cap E_{N} .
\end{aligned}
$$

This implies that $y \in \operatorname{int}_{E_{N}}\left(G^{-1}(\bar{x}(y)) \cap E_{N}\right)$. Thus, for every $N \in\langle E\rangle$, there exists a nonempty compact convex subset $E_{N}$ of $E$ containing $N$ such that

$$
E_{N} \backslash K \subseteq \bigcup_{x \in E_{N}} \operatorname{int}_{E_{N}}\left(G^{-1}(x) \cap E_{N}\right)
$$

Moreover, if (iv) $)_{2}$ is satisfied, then there exists a point $x_{0}=\left(x_{0 i}\right)_{i \in I} \in E$ such that

$$
\operatorname{cl}_{E}\left(E \backslash G^{-1}\left(x_{0}\right)\right)=\operatorname{cl}_{E}\left(E \backslash \bigcap_{i \in I} G_{i}^{-1}\left(x_{0 i}\right)\right) \subseteq K
$$

Therefore, by Theorem 3.1, there exists $\hat{y}=\left(\hat{y}_{i}\right)_{i \in I} \in E$ such that $\hat{y} \in G(\hat{y})$; that is, $\hat{y}_{i} \in G_{i}(\hat{y})$ for every $i \in I$. This completes the proof.

Remark 3.7 We can compare Theorem 3.4 with Theorem 3 of Prokopovych [35] in the following aspects: (1) every $E_{i}$ in Theorem 3.4 does not need to be compact and it does not possess any linear structure; (2) in Theorem 3.4, there are two set-valued mappings, 
but there is only one set-valued mapping in Theorem 3 of Prokopovych [35]; (3) (iii) of Theorem 3.4 is weaker than the corresponding condition of Theorem 3 of Prokopovych [35] because the domain of every $F_{i}$ does not need to be $E$.

\section{Minimax inequalities with applications}

In this section, by using Theorem 3.1, we will give minimax inequalities in noncompact $\mathrm{CAT}(0)$ spaces. As applications of minimax inequalities, we obtain a saddle point theorem, a fixed point theorem for single-valued mappings, and best approximation theorems in the setting of noncompact $\mathrm{CAT}(0)$ spaces.

Theorem 4.1 Let $(E, d)$ be a complete CAT(0) space with the convex hull finite property, $K$ be a nonempty compact subset of $E$, and $f, g: E \times E \rightarrow \mathbb{R} \cup\{ \pm \infty\}$ be two functions such that

(i) for every $(x, y) \in E \times E, f(x, y) \leq g(x, y)$;

(ii) for every $y \in E$, the set $\{x \in E: g(x, y)>0\}$ is convex;

(iii) for every $x \in E, y \mapsto f(x, y)$ is lower semicontinuous on $E$;

(iv) for every $y \in E, g(y, y) \leq 0$;

(v) one of the following conditions holds:

$(\mathrm{v})_{1}$ for every $N \in\langle E\rangle$, there exists a nonempty compact convex subset $E_{N}$ of $E$ containing $N$ such that

$$
E_{N} \backslash K \subseteq \bigcup_{x \in E_{N}} \operatorname{int}_{E_{N}}\left(\{y \in E: g(x, y)>0\} \cap E_{N}\right) ;
$$

$(\mathrm{v})_{2}$ there exists a point $x_{0} \in E$ such that $\operatorname{cl}_{E}\left(E \backslash\left\{y \in E: g\left(x_{0}, y\right)>0\right\}\right) \subseteq K$.

Then there exists $\hat{y} \in K$ such that $f(x, \hat{y}) \leq 0$ for every $x \in E$.

Proof Define two set-valued mappings $F, G: E \rightarrow 2^{E}$ by

$$
F(y)=\{x \in E: f(x, y)>0\} \quad \text { and } \quad G(y)=\{x \in E: g(x, y)>0\}, \quad y \in E .
$$

By (i) and (ii), $F(y) \subseteq G(y)$ and $G(y)$ is convex for every $y \in E$. By (iii), $F^{-1}(x)$ is open in $E$ for every $x \in E$. It follows from (v) and the definition of $G$ that one of the following conditions holds:

(a) for every $N \in\langle E\rangle$, there exists a nonempty compact convex subset $E_{N}$ of $E$ containing $N$ such that

$$
E_{N} \backslash K \subseteq \bigcup_{x \in E_{N}} \operatorname{int}_{E_{N}}\left(G^{-1}(x) \cap E_{N}\right) ;
$$

(b) there exists a point $x_{0} \in E$ such that $\operatorname{cl}_{E}\left(E \backslash G^{-1}\left(x_{0}\right)\right) \subseteq K$.

By (iv), $y \notin G(y)$ for every $y \in E$, which implies that the conclusion of Theorem 3.1 does not hold. Hence, (iii) of Theorem 3.1 is not true. So, there exists $\hat{y} \in K$ such that $F(\hat{y})=\emptyset$, which implies that $f(x, \hat{y}) \leq 0$ for every $x \in E$. This completes the proof.

Remark 4.1 If $f=g$, then $(\mathrm{v})_{1}$ and $(\mathrm{v})_{2}$ of Theorem 4.1 can be replaced by the following equivalent conditions, respectively: 
$(\mathrm{v})_{1}^{\prime}$ for every $N \in\langle E\rangle$, there exists a nonempty compact convex subset $E_{N}$ of $E$ containing $N$ such that $E_{N} \backslash K \subseteq \bigcup_{x \in E_{N}}\{y \in E: f(x, y)>0\}$;

$(\mathrm{v})_{2}^{\prime}$ there exists a point $x_{0} \in E$ such that $E \backslash\left\{y \in E: f\left(x_{0}, y\right)>0\right\} \subseteq K$.

Remark 4.2 (ii) of Theorem 4.1 can be replaced by the following condition:

(ii)' Let $y \in E$ be given. For every $x_{1}, x_{2} \in E$ and every $t \in[0,1]$, we have

$$
g\left((1-t) x_{1} \oplus t x_{2}, y\right) \geq \min \left\{g\left(x_{1}, y\right), g\left(x_{2}, y\right)\right\} .
$$

In fact, we can show that (ii)' implies (ii) of Theorem 4.1. Suppose to the contrary that (ii) of Theorem 4.1 does not hold; that is, for every given $y \in E$, there exist $x_{1}, x_{2} \in\{x \in E: g(x, y)>$ $0\}$ and the unique geodesic $\gamma$ joining $x_{1}$ and $x_{2}$ such that $\left[x_{1}, x_{2}\right] \nsubseteq\{x \in E: g(x, y)>0\}$ and $x_{1} \neq x_{2}$. By Lemma 2.6, there exists $t_{0} \in[0,1]$ such that $\left(1-t_{0}\right) x_{1} \oplus t_{0} x_{2} \notin\{x \in E: g(x, y)>0\}$. Therefore, by (ii)' and by the fact that $\left[x_{1}, x_{2}\right] \nsubseteq\{x \in E: g(x, y)>0\}$, we have

$$
0 \geq g\left(\left(1-t_{0}\right) x_{1} \oplus t_{0} x_{2}, y\right) \geq \min \left\{g\left(x_{1}, y\right), g\left(x_{2}, y\right)\right\}>0,
$$

which is a contraction. Hence, (ii) of Theorem 4.1 holds.

Remark 4.3 Theorem 3.1 is equivalent to Theorem 4.1. We have shown that Theorem 3.1 implies Theorem 4.1. Now we show that Theorem 4.1 implies Theorem 3.1. Suppose that all the hypotheses of Theorem 3.1 are satisfied. Define two real-valued functions $f, g: E \times$ $E \rightarrow \mathbb{R}$ by

$$
\begin{aligned}
& f(x, y)= \begin{cases}1, & x \in F(y), \\
0, & x \notin F(y),\end{cases} \\
& g(x, y)= \begin{cases}1, & x \in G(y), \\
0, & x \notin G(y) .\end{cases}
\end{aligned}
$$

By (i) of Theorem 3.1, for every $(x, y) \in E \times E, f(x, y) \leq g(x, y)$ and the set $\{x \in E: g(x, y)>0\}$ is convex for every $y \in E$. For every $x \in E$ and every $r \in \mathbb{R}$, we have

$$
\{y \in E: f(x, y)>r\}= \begin{cases}E, & r<0, \\ \emptyset, & r \geq 1, \\ F^{-1}(x), & 0 \leq r<1 .\end{cases}
$$

Hence, by (ii) of Theorem 3.1, for every $x \in E$ and every $r \in \mathbb{R}$, the set $\{y \in E: f(x, y)>r\}$ is open in $E$, which implies that for every $x \in E$, the function $y \mapsto f(x, y)$ is lower semicontinuous on $E$. If the conclusion of Theorem 3.1 were not true, then, for every $y \in E$, we have $g(y, y) \leq 0$. Suppose that (iv) $)_{1}$ of Theorem 3.1 holds. Then, by (iv) $)_{1}$ of Theorem 3.1 and by the definition of $g$, we know that, for every $N \in\langle E\rangle$, there exists a nonempty compact convex subset $E_{N}$ of $E$ containing $N$ such that

$$
E_{N} \backslash K \subseteq \bigcup_{x \in E_{N}} \operatorname{int}_{E_{N}}\left(\{y \in E: g(x, y)>0\} \cap E_{N}\right) .
$$


If (iv) $)_{2}$ of Theorem 3.1 holds, then, by (iv) $)_{2}$ of Theorem 3.1 and by the definition of $g$, there exists a point $x_{0} \in E$ such that $\operatorname{cl}_{E}\left(E \backslash\left\{y \in E: g\left(x_{0}, y\right)>0\right\}\right) \subseteq K$. Thus, all the hypotheses of Theorem 4.1 are satisfied. By Theorem 4.1, there exists $\hat{y} \in K$ such that $f(x, \hat{y}) \leq 0$ for every $x \in E$. Therefore, $x \notin F(\hat{y})$ for every $x \in E$, which implies that $F(\hat{y})=\emptyset$. This contradicts (iii) of Theorem 3.1. Hence, the conclusion of Theorem 3.1 must hold.

Remark 4.4 Theorem 4.1 generalizes Theorem 5.3 of Yang and $\mathrm{Pu}$ [34] in the following aspects: (1) The underlying spaces of Theorem 4.1 and Theorem 5.3 of Yang and $\mathrm{Pu}$ [34] are $\mathrm{CAT}(0)$ spaces and Hadamard manifolds, respectively. We can see that $\mathrm{CAT}(0)$ spaces include Hadamard manifolds as special cases (see [23]); (2) the $E$ in Theorem 4.1 does not need to be compact; (3) in Theorem 4.1, there are two functions, but there is only one function in Theorem 5.3 of Yang and Pu [34].

Remark 4.5 By Remarks 3.5 and 4.3, we know that Theorem 3.1, Theorem 3.3 and Theorem 4.1 are equivalent.

Corollary 4.1 Let $C$ be a closed convex subset of a complete CAT(0) space $(E, d)$ with the convex hull finite property, $K$ be a nonempty compact subset of $C$, and $f, g: C \times C \rightarrow \mathbb{R} \cup$ $\{ \pm \infty\}$ be two functions. Suppose that $\sup _{y \in C} g(y, y)<+\infty$ and the following conditions are fulfilled:

(i) for every $(x, y) \in C \times C, f(x, y) \leq g(x, y)$;

(ii) for every $y \in C$, the set $\left\{x \in C: g(x, y)>\sup _{y \in C} g(y, y)\right\}$ is convex;

(iii) for every $x \in C, y \mapsto f(x, y)$ is lower semicontinuous on $C$;

(iv) one of the following conditions holds:

(iv) ${ }_{1}$ for every $N \in\langle C\rangle$, there exists a nonempty compact convex subset $C_{N}$ of $C$ containing $N$ such that

$$
C_{N} \backslash K \subseteq \bigcup_{x \in C_{N}} \operatorname{int}_{C_{N}}\left(\left\{y \in C: g(x, y)>\sup _{y \in C} g(y, y)\right\} \cap C_{N}\right) ;
$$

(iv) $)_{2}$ there exists a point $x_{0} \in C$ such that

$$
\operatorname{cl}_{C}\left(C \backslash\left\{y \in C: g\left(x_{0}, y\right)>\sup _{y \in C} g(y, y)\right\}\right) \subseteq K .
$$

Then there exists $\hat{y} \in K$ such that $f(x, \hat{y}) \leq \sup _{y \in C} g(y, y)$ for every $x \in C$.

Proof Since $C$ is a closed convex subset of the complete CAT $(0)$ space $(E, d)$ with the convex finite property, it follows that $C$ equipped with the induced metric is also a complete $\mathrm{CAT}(0)$ space with the convex hull finite property. Define two functions $f^{\prime}, g^{\prime}: C \times C \rightarrow$ $\mathbb{R} \cup\{ \pm \infty\}$ by

$$
\begin{array}{ll}
f^{\prime}(x, y)=f(x, y)-\sup _{y \in C} g(y, y), & (x, y) \in C \times C, \\
g^{\prime}(x, y)=g(x, y)-\sup _{y \in C} g(y, y), & (x, y) \in C \times C .
\end{array}
$$

We can easily check that $f^{\prime}, g^{\prime}$ satisfy all the hypotheses of Theorem 4.1. Therefore, by Theorem 4.1, we infer that there exists $\hat{y} \in K$ such that $f^{\prime}(x, \hat{y}) \leq 0$ for every $x \in C$; that is, $f(x, \hat{y}) \leq \sup _{y \in C} g(y, y)$ for every $x \in C$. This completes the proof. 
Remark 4.6 Corollary 4.1 generalizes Theorem 3.3 of Shabanian and Vaezpour [18] in the following aspects: (1) the set $C$ in Corollary 4.1 does not need to be compact; (2) (ii) of Corollary 4.1 is weaker than the corresponding (2) of Theorem 3.3 of Shabanian and Vaezpour [18]; (3) in Corollary 4.1, there are two functions, but there is only one function in Theorem 3.3 of Shabanian and Vaezpour [18].

By Theorem 4.1, we get the following saddle point theorem in CAT(0) spaces.

Theorem 4.2 Let $(E, d)$ be a complete CAT(0) space with the convex hull finite property, $K_{1}, K_{2} \subseteq E$ be two nonempty compact sets, and $f: E \times E \rightarrow \mathbb{R} \cup\{ \pm \infty\}$ be a real-valued continuous function. Assume that

(i) for every $y \in E, f(y, y)=0$;

(ii) for every $y \in E$, the set $\{x \in E: f(x, y)>0\}$ is convex;

(iii) for every $x \in E$, the set $\{y \in E: f(x, y)<0\}$ is convex;

(iv) one of the following conditions holds:

(iv) ${ }_{1}$ for every $N \in\langle E\rangle$, there exist two nonempty compact convex subsets $E_{N}, \widetilde{E}_{N}$ of E containing $N$ such that

$$
E_{N} \backslash K_{1} \subseteq \bigcup_{x \in E_{N}}\{y \in E: f(x, y)>0\}
$$

and

$$
\widetilde{E}_{N} \backslash K_{2} \subseteq \bigcup_{y \in \widetilde{E}_{N}}\{x \in E: f(x, y)<0\} ;
$$

(iv) 2 there exist two points $x_{0}, y_{0} \in E$ such that

$$
E \backslash K_{1} \subseteq\left\{y \in E: f\left(x_{0}, y\right)>0\right\} \quad \text { and } \quad E \backslash K_{2} \subseteq\left\{x \in E: f\left(x, y_{0}\right)<0\right\}
$$

Then $f$ has a saddle point $(\hat{x}, \hat{y}) \in K_{1} \times K_{2}$; that is, $f(x, \hat{y}) \leq f(\hat{x}, \hat{y}) \leq f(\hat{x}, y)$ for every $(x, y) \in$ $E \times E$. In particular, $\inf _{x \in E} \sup _{y \in E} f(x, y)=\sup _{y \in E} \inf _{x \in E} f(x, y)$.

Proof By (i), (ii), the continuity of $f$, the first parts of (iv) ${ }_{1}$ and (iv) $)_{2}$, and Remark 4.1, we can see that all the conditions of Theorem 4.1 with $f=g$ are satisfied. Thus, by Theorem 4.1 with $f=g$, there exists $\hat{y} \in K_{1}$ such that $f(x, \hat{y}) \leq 0$ for every $x \in E$. Let $f^{\prime}: E \times E \rightarrow \mathbb{R} \cup$ $\{ \pm \infty\}$ be defined by $f^{\prime}(y, x)=-f(x, y)$ for every $(y, x) \in E \times E$. Then by (i), (iii), the continuity of $f$, the second parts of (iv) $)_{1}$ and (iv) $)_{2}$, and Remark 4.1, we can see that all the hypotheses of Theorem 4.1 with $f=g$ are fulfilled. Hence, by Theorem 4.1 with $f=g$, there exists $\hat{x} \in K_{2}$ such that $f^{\prime}(y, \hat{x}) \leq 0$ for every $y \in E$. Therefore, we get

$$
f(x, \hat{y}) \leq 0=f(\hat{x}, \hat{y}) \leq f(\hat{x}, y) \quad \text { for all }(x, y) \in E \times E,
$$

and so

$$
\inf _{x \in E} \sup _{y \in E} f(x, y) \leq \sup _{y \in E} \inf _{x \in E} f(x, y)
$$


Since $\inf _{x \in E} \sup _{y \in E} f(x, y) \geq \sup _{y \in E} \inf _{x \in E} f(x, y)$ is always true, we have

$$
\inf _{x \in E} \sup _{y \in E} f(x, y)=\sup _{y \in E} \inf _{x \in E} f(x, y) .
$$

This completes the proof.

By Theorem 4.1, we have the following best approximation theorem in CAT(0) spaces.

Theorem 4.3 Let $(E, d)$ be a complete CAT(0) space, $C \subseteq E$ be a closed locally compact convex set, $H: C \rightarrow E$ be a continuous mapping. Suppose that there exists a nonempty compact subset $K$ of $C$ such that one of the following conditions holds:

(i) 1 for every $N \in\langle C\rangle$, there exists a nonempty compact convex subset $C_{N}$ of $C$ containing $N$ such that

$$
C_{N} \backslash K \subseteq \bigcup_{x \in C_{N}}\{y \in C: d(y, H(y))>d(x, H(y))\}
$$

(i) 2 there exists a point $x_{0} \in C$ such that

$$
C \backslash\left\{y \in C: d(y, H(y))>d\left(x_{0}, H(y)\right)\right\} \subseteq K
$$

Then there exists $\hat{y} \in K$ such that $d(\hat{y}, H(\hat{y}))=\inf _{x \in C} d(x, H(\hat{y}))$.

Proof Since $C$ is a closed locally compact convex subset of $E$, it follows that $C$ with the induced metric is a complete locally compact CAT(0) space. By Lemma 2.4, $C$ has the convex hull finite property. Define a function $f: C \times C \rightarrow \mathbb{R}$ by

$$
f(x, y)=d(y, H(y))-d(x, H(y)), \quad(x, y) \in C \times C .
$$

Since $H$ is continuous, it is evident that, for every $x \in C$, the function $y \mapsto d(y, H(y))-$ $d(x, H(y))$ is lower semicontinuous. For every $y \in C, f(y, y)=0$. By the assumption and by the definition of $f$, we know that one of the following conditions holds:

(a) for every $N \in\langle C\rangle$, there exists a nonempty compact convex subset $C_{N}$ of $C$ containing $N$ such that $C_{N} \backslash K \subseteq \bigcup_{x \in C_{N}}\{y \in C: f(x, y)>0\}$;

(b) there exists a point $x_{0} \in C$ such that $C \backslash\left\{y \in C: f\left(x_{0}, y\right)>0\right\} \subseteq K$.

It remains to prove that for every fixed $y \in C$, the set $\{x \in C: f(x, y)>0\}$ is convex. Suppose to the contrary that there exist two points $x_{1}, x_{2} \in\{x \in C: f(x, y)>0\}$, the unique geodesic $\gamma:[0, l] \rightarrow C$ jointing $x_{1}, x_{2}$, and $t_{0} \in[0, l]$ such that $\gamma\left(t_{0}\right) \notin\{x \in C: f(x, y)>0\}$, which implies that $d(y, H(y)) \leq d\left(\gamma\left(t_{0}\right), H(y)\right)$. Since $x_{1}, x_{2} \in\{x \in C: f(x, y)>0\}$, it follows that

$$
d\left(x_{1}, H(y)\right)<d(y, H(y)) \text { and } d\left(x_{2}, H(y)\right)<d(y, H(y)) .
$$

Hence, we have

$$
x_{1} \in U(H(y), d(y, H(y))) \text { and } \quad x_{2} \in U(H(y), d(y, H(y))) \text {, }
$$


where $U(H(y), d(y, H(y)))$ denotes the open ball centered at $H(y)$ with radius $d(y, H(y))$. Since every ball in the $\operatorname{CAT}(0)$ space $(E, d)$ is convex (see [23]), it follows that

$$
\gamma([0, l]) \subseteq U(H(y), d(y, H(y))),
$$

which implies that $\gamma\left(t_{0}\right) \in U(H(y), d(y, H(y)))$; that is, $d(y, H(y))>d\left(\gamma\left(t_{0}\right), H(y)\right)$. This contradicts $d(y, H(y)) \leq d\left(\gamma\left(t_{0}\right), H(y)\right)$. Therefore, for every $y \in C$, the set $\{x \in C: f(x, y)>0\}$ is convex. Thus, by Remark 4.1, all the requirements of Theorem 4.1 with $f=g$ are fulfilled. Hence, by Theorem 4.1 with $f=g$, there exists $\hat{y} \in K$ such that $f(x, \hat{y}) \leq 0$ for every $x \in C$; that is, $d(\hat{y}, H(\hat{y})) \leq d(x, H(\hat{y}))$ for every $x \in C$, which implies that $d(\hat{y}, H(\hat{y}))=$ $\inf _{x \in C} d(x, H(\hat{y}))$. This completes the proof.

Remark 4.7 Theorem 4.3 generalizes Theorem 3.1 of Shabanian and Vaezpour [18] in the following aspects: (1) the $C$ in Theorem 4.3 does not need to be compact; (2) the $E$ in Theorem 4.3 does not need to have the convex hull finite property. We point out that the proof of Theorem 4.3 is different from that of Theorem 3.1 of Shabanian and Vaezpour [18].

As an application of Theorem 4.3, we have the following fixed point theorem for singlevalued mappings.

Theorem 4.4 Let $(E, d)$ be a complete $\mathrm{CAT}(0)$ space, $C \subseteq E$ be a closed locally compact convex set, $K$ be a nonempty compact subset of $C$, and $H: C \rightarrow E$ be a continuous mapping such that

(i) for every $c \in K$ with $c \neq H(c)$, there exists $t \in(0,1)$ such that

$$
C \cap U(H(c),(1-t) d(c, H(c))) \neq \emptyset,
$$

where $U(H(c),(1-t) d(c, H(c)))$ denotes the open ball centered at $H(c)$ with radius $(1-t) d(c, H(c))$;

(ii) one of the following conditions holds:

(ii) $)_{1}$ for every $N \in\langle C\rangle$, there exists a nonempty compact convex subset $C_{N}$ of $C$ containing $N$ such that

$$
C_{N} \backslash K \subseteq \bigcup_{x \in C_{N}}\{y \in C: d(y, H(y))>d(x, H(y))\}
$$

(ii) $)_{2}$ there exists a point $x_{0} \in C$ such that

$$
C \backslash\left\{y \in C: d(y, H(y))>d\left(x_{0}, H(y)\right)\right\} \subseteq K
$$

Then there exists $\hat{y} \in K$ such that $\hat{y}=H(\hat{y})$.

Proof It follows from Theorem 4.3 that there exists a point $\hat{y} \in K$ such that $d(\hat{y}, H(\hat{y}))=$ $\inf _{x \in C} d(x, H(\hat{y}))$. We show that $\hat{y}$ is a fixed point of $H$. Suppose not. Then by (i), there exists $t \in(0,1)$ such that $C \cap U(H(\hat{y}),(1-t) d(\hat{y}, H(\hat{y}))) \neq \emptyset$. Take $\hat{x} \in C \cap U(H(\hat{y}),(1-t) d(\hat{y}, H(\hat{y})))$. 
Then we have $\hat{x} \neq \hat{y}$ and

$$
d(\hat{x}, H(\hat{y}))<(1-t) d(\hat{y}, H(\hat{y}))<d(\hat{y}, H(\hat{y}))
$$

which contradicts the fact that $d(\hat{y}, H(\hat{y}))=\inf _{x \in C} d(x, H(\hat{y}))$. Therefore, $\hat{y}$ is a fixed point of $H$. This completes the proof.

Remark 4.8 Theorem 4.4 generalizes Theorem 3.2 of Shabanian and Vaezpour [18] in the following aspects: (1) the $E$ in Theorem 4.4 does not need to have the convex hull finite property; (2) the $C$ in Theorem 4.4 does not need to be compact.

By Theorem 4.1, we obtain the following generalized best approximation theorem.

Theorem 4.5 Let $(E, d)$ be a complete $\mathrm{CAT}(0)$ space, $C \subseteq E$ be a closed locally compact convex set, $K$ be a nonempty compact subset of $C$, and $G, H: C \rightarrow 2^{E}$ be two upper semicontinuous set-valued mappings with nonempty compact values. Assume that

(i) for every $y \in C$, the set $\{x \in C: d(G(y), H(y))>d(G(x), H(y))\}$ is convex;

(ii) one of the following conditions holds:

(ii) $)_{1}$ for every $N \in\langle C\rangle$, there exists a nonempty compact convex subset $C_{N}$ of $C$ containing $N$ such that

$$
C_{N} \backslash K \subseteq \bigcup_{x \in C_{N}}\{y \in C: d(G(y), H(y))>d(G(x), H(y))\} ;
$$

(ii) $)_{2}$ there exists a point $x_{0} \in C$ such that

$$
C \backslash\left\{y \in C: d(G(y), H(y))>d\left(G\left(x_{0}\right), H(y)\right)\right\} \subseteq K .
$$

Then there exists $\hat{y} \in K$ such that $d(G(\hat{y}), H(\hat{y}))=\inf _{x \in C} d(G(x), H(\hat{y}))$.

Proof Since $C$ is a closed locally compact convex subset of $E$, we know that $C$ with the induced metric is a complete locally compact $\mathrm{CAT}(0)$ space. So, by Lemma $2.4, C$ has the convex hull finite property. Define a function $f: C \times C \rightarrow \mathbb{R}$ by

$$
f(x, y)=d(G(y), H(y))-d(G(x), H(y)), \quad(x, y) \in C \times C .
$$

In order to prove that the function $y \mapsto f(x, y)$ is lower semicontinuous for every $x \in C$, it suffices to show that, for every $x \in C$ and for every $r \in \mathbb{R}$, the set $\{y \in C: f(x, y) \leq r\}$ is closed. Let $x \in C, r \in \mathbb{R}$ be fixed and let $\left\{y_{n}\right\}_{n \geq 1} \subseteq\{y \in C: f(x, y) \leq r\}$ be an arbitrary sequence such that $y_{n} \rightarrow y^{*} \in C$ as $n \rightarrow \infty$. Let $\varepsilon>0$ be arbitrary. Since $G$ is an upper semicontinuous set-valued mapping with nonempty compact values, it follows from the result of Aubin and Frankowska [36, p.39] that there exists $\eta>0$ such that for every $y^{\prime} \in$ $U\left(y^{*}, \eta\right), G\left(y^{\prime}\right) \subseteq U\left(G\left(y^{*}\right), \frac{1}{3} \varepsilon\right)=\bigcup_{x \in G\left(y^{*}\right)} U\left(x, \frac{1}{3} \varepsilon\right)$, where $U\left(y^{*}, \eta\right)$ and $U\left(x, \frac{1}{3} \varepsilon\right)$ denote the open ball centered at $y^{*}$ with radius $\eta$ and the open ball centered at $x$ with radius $\frac{1}{3} \varepsilon$, respectively. By the convergence of sequence $\left\{y_{n}\right\}_{n \geq 1}$, we know that there exists $N_{1} \in \mathbb{N}$ such that for every $n \geq N_{1}$, we have $y_{n} \in U\left(y^{*}, \eta\right)$ and thus, $G\left(y_{n}\right) \subseteq U\left(G\left(y^{*}\right), \frac{1}{3} \varepsilon\right)$. Similarly, 
we can prove that there exists $N_{2} \in \mathbb{N}$ such that for every $n \geq N_{2}, H\left(y_{n}\right) \subseteq U\left(H\left(y^{*}\right), \frac{1}{3} \varepsilon\right)$. Now we let $N=\max \left\{N_{1}, N_{2}\right\}$. Then, for every $n \geq N$, we have the following:

$$
\begin{aligned}
d\left(G\left(y^{*}\right), H\left(y^{*}\right)\right) & \leq d\left(G\left(y^{*}\right), G\left(y_{n}\right)\right)+d\left(G\left(y_{n}\right), H\left(y_{n}\right)\right)+d\left(H\left(y_{n}\right), H\left(y^{*}\right)\right) \\
& <\frac{2}{3} \varepsilon+d\left(G\left(y_{n}\right), H\left(y_{n}\right)\right) \\
& \leq \frac{2}{3} \varepsilon+r+d\left(G(x), H\left(y_{n}\right)\right) \\
& \leq \frac{2}{3} \varepsilon+r+d\left(G(x), H\left(y^{*}\right)\right)+d\left(H\left(y^{*}\right), H\left(y_{n}\right)\right) \\
& <\varepsilon+r+d\left(G(x), H\left(y^{*}\right)\right) .
\end{aligned}
$$

By the arbitrariness of $\varepsilon$, we have $d\left(G\left(y^{*}\right), H\left(y^{*}\right)\right)-d\left(G(x), H\left(y^{*}\right)\right) \leq r$, which implies that $y^{*} \in\{y \in C: f(x, y) \leq r\}$ and thus, the set $\{y \in C: f(x, y) \leq r\}$ is closed. Therefore, for every $x \in C$, the function $y \mapsto f(x, y)$ is lower semicontinuous. For every $y \in C$, we have $f(y, y)=0$. By (i), for every $y \in C$, the set $\{x \in C: f(x, y)>0\}$ is convex. By the assumption and by the definition of $f$, we know that one of the following conditions holds:

(a) for every $N \in\langle C\rangle$, there exists a nonempty compact convex subset $C_{N}$ of $C$ containing $N$ such that $C_{N} \backslash K \subseteq \bigcup_{x \in C_{N}}\{y \in C: f(x, y)>0\}$;

(b) there exists a point $x_{0} \in C$ such that $C \backslash\left\{y \in C: f\left(x_{0}, y\right)>0\right\} \subseteq K$.

By Remark 4.1, all the requirements of Theorem 4.1 with $f=g$ are satisfied. Hence, by Theorem 4.1 with $f=g$, there exists $\hat{y} \in K$ such that $f(x, \hat{y}) \leq 0$ for every $x \in C$; that is, $d(G(\hat{y}), H(\hat{y})) \leq d(G(x), H(\hat{y}))$ for every $x \in C$, which implies that $d(G(\hat{y}), H(\hat{y}))=$ $\inf _{x \in C} d(G(x), H(\hat{y}))$. This completes the proof.

Remark 4.9 Theorem 4.5 generalizes Theorem 3.4 of Shabanian and Vaezpour [18] in the following aspects: (1) the $C$ in Theorem 4.5 does not need to be compact; (2) the $E$ in Theorem 4.5 does not need to have the convex hull finite property; (3) the set-valued mappings $G, H$ in Theorem 4.5 do not need to have convex values; (4) the condition that the set-valued mapping $G$ in Theorem 3.4 of Shabanian and Vaezpour [18] is quasi-convex is removed. We point out that the proof of Theorem 4.5 is different from that of Theorem 3.4 of Shabanian and Vaezpour [18].

Remark 4.10 Theorem 4.5 can be regarded as a generalization of Theorem 4.3. In fact, let $G(y)=\{y\}$ for every $y \in C$ and $H$ be a single-valued continuous mapping. Then by using the same method as in the proof Theorem 4.3, we can show that (i) of Theorem 4.5 holds and thus, Theorem 4.5 reduces to Theorem 4.3 .

\section{Existence of $\boldsymbol{\varphi}$-equilibrium for multiobjective games}

In this section, we will consider the multiobjective noncooperative game in its strategic form $\Gamma=\left(X_{i}, V^{i}\right)_{i \in I}$, where $I=\{1,2, \ldots, n\}$ is the set of players; every $X_{i}$ is the strategy set of the $i$ th player and every $V^{i}: X=\prod_{i \in I} X_{i} \rightarrow \mathbb{R}^{k_{i}}$ is the payoff function of the $i$ th player with $k_{i}$ being a positive integer. If an action combination $x=\left(x_{1}, x_{2}, \ldots, x_{n}\right)$ is played, every player $i$ is trying to confirm his/her vector payoff function $V^{i}(x):=\left(f_{1}^{i}(x), f_{2}^{i}(x), \ldots, f_{k_{i}}^{i}(x)\right)$ and then minimize his/her vector payoff function according to his/her preference.

Before we introduce the equilibrium concepts of multiobjective noncooperative games, we give the following notation. 
For every $m \in \mathbb{N}$, let $\mathbb{R}_{+}^{m}:=\left\{q:=\left(q_{1}, q_{2}, \ldots, q_{m}\right) \in \mathbb{R}^{m}: q_{j} \geq 0, \forall j=1,2, \ldots, m\right\}$ and $\operatorname{int}_{\mathbb{R}^{m}} \mathbb{R}_{+}^{m}:=\left\{q:=\left(q_{1}, q_{2}, \ldots, q_{m}\right) \in \mathbb{R}^{m}: q_{j}>0, \forall j=1,2, \ldots, m\right\}$ denote the nonnegative orthant of $\mathbb{R}^{m}$ and the nonempty interior of $\mathbb{R}_{+}^{m}$ with the Euclidian metric topology, respectively. For every $q, r \in \mathbb{R}^{m}$, let $q \cdot r$ denote the standard Euclidean inner product.

We denote $X_{\hat{i}}:=\prod_{j \in I \backslash i} X_{j}$ for every $i \in I$. If $x=\left(x_{1}, x_{2}, \ldots, x_{n}\right) \in X$, then we write $x_{\hat{i}}=$ $\left(x_{1}, \ldots, x_{i-1}, x_{i+1}, \ldots, x_{n}\right)$ for every $i \in I$. We use the following notation

$$
\begin{aligned}
& \left(x_{\hat{i}}, y_{i}\right):=\left(x_{1}, x_{2}, \ldots, x_{i-1}, y_{i}, x_{i+1}, \ldots, x_{n}\right) \in X \quad \text { and } \\
& \left(x_{\hat{i}}, x_{i}\right):=\left(x_{1}, x_{2}, \ldots, x_{i-1}, x_{i}, x_{i+1}, \ldots, x_{n}\right)=x \in X .
\end{aligned}
$$

Let $\hat{x}=\left(\hat{x}_{1}, \hat{x}_{2}, \ldots, \hat{x}_{n}\right) \in X$ and let $\varphi=\left(\varphi_{1}, \varphi_{2}, \ldots, \varphi_{n}\right): X \rightarrow X$ be a surjective mapping defined by

$$
\varphi(x)=\left(\varphi_{1}\left(x_{1}\right), \varphi_{1}\left(x_{2}\right), \ldots, \varphi_{n}\left(x_{n}\right)\right), \quad x=\left(x_{1}, x_{2}, \ldots, x_{n}\right) \in X .
$$

Now we introduce the following definitions.

Definition 5.1 A strategy $\hat{x}_{i} \in X_{i}$ of player $i$ is said to be a Pareto efficient $\varphi$-strategy (respectively, a weak Pareto efficient $\varphi$-strategy) with respect to $\hat{x}$ if there is no strategy $x_{i} \in X_{i}$ such that

$$
V^{i}(\hat{x})-V^{i}\left(\hat{x}_{\hat{i}}, \varphi\left(x_{i}\right)\right) \in \mathbb{R}_{+}^{k_{i}} \backslash\{0\} \quad\left(\text { respectively, } V^{i}(\hat{x})-V^{i}\left(\hat{x}_{\hat{i}}, \varphi\left(x_{i}\right)\right) \in \operatorname{int}_{\mathbb{R}^{k_{i}}} \mathbb{R}_{+}^{k_{i}}\right)
$$

Definition 5.2 A strategy $\hat{x} \in X$ is said to be a Pareto $\varphi$-equilibrium (respectively, a weak Pareto $\varphi$-equilibrium) of a game $\Gamma=\left(X_{i}, V^{i}\right)_{i \in I}$ if, for every $i \in I, \hat{x}_{i} \in X_{i}$ is a Pareto efficient $\varphi$-strategy (respectively, a weak Pareto efficient $\varphi$-strategy) with respect to $\hat{x}$.

Remark 5.1 Definitions 5.1-5.2 generalize the corresponding definitions of Wang [37], Yuan and Tarafdar [38], and Yu and Yuan [39]. In fact, if $\varphi_{i}\left(x_{i}\right)=x_{i}$ for every $x=\left(x_{i}\right)_{i \in I} \in X$ and every $i \in I$, then Definitions 5.1-5.2 coincide with the corresponding definitions of Wang [37], Yuan and Tarafdar [38], and Yu and Yuan [39]. By the above definition, we can see that every Pareto $\varphi$-equilibrium is a weak Pareto $\varphi$-equilibrium, but the converse is not true in general.

Definition 5.3 A strategy $\hat{x} \in X$ is said to be a weighted Nash $\varphi$-equilibrium with respect to the weighted vector $Q:=\left(Q_{1}, Q_{2}, \ldots, Q_{n}\right)$ of a game $\Gamma=\left(X_{i}, V^{i}\right)_{i \in I}$ if, for every $i \in I$, we have

(i) $Q_{i}=\left(Q_{i, 1}, Q_{i, 2}, \ldots, Q_{i, k_{i}}\right) \in \mathbb{R}_{+}^{k_{i}} \backslash\{0\}$;

(ii) $Q_{i} \cdot V^{i}(\hat{x}) \leq Q_{i} \cdot V^{i}\left(\hat{x}_{\hat{i}}, \varphi\left(x_{i}\right)\right)$ for every $x_{i} \in X_{i}$.

Remark 5.2 If $\varphi_{i}\left(x_{i}\right)=x_{i}$ for every $x=\left(x_{i}\right)_{i \in I} \in X$ and every $i \in I$, then Definition 5.3 reduces to Definition 2.3 of Wang [37] and Definition 3 of Yuan and Tarafdar [38] and Yu and Yuan [39]. In particular, if $Q_{i} \in \mathbb{R}_{+}^{k_{i}}$ with $\sum_{j=1}^{k_{i}} Q_{i, j}=1$ for every $i \in I$, then the strategy $\hat{x} \in X$ is said to be a normalized weighted Nash $\varphi$-equilibrium with respect to $Q$.

As an application of Theorem 4.1, we have the following existence theorem of weighted Nash $\varphi$-equilibrium for multiobjective noncooperative games in the setting of noncompact $\mathrm{CAT}(0)$ spaces. 
Theorem 5.1 Let $\Gamma=\left(X_{i}, V^{i}\right)_{i \in I}$ be a multiobjective game with $V^{i}=\left(f_{1}^{i}, f_{2}^{i}, \ldots, f_{k_{i}}^{i}\right)$. For every $i \in I$, let $X_{i}$ be a nonempty subset of a $\mathrm{CAT}(0)$ space $\left(E_{i}, d_{i}\right)$ such that $X=\prod_{i \in I} X_{i}$ is a complete $\mathrm{CAT}(0)$ space with the convex hull finite property. Let $K$ be a nonempty compact subset of $X$ and $\varphi=\left(\varphi_{1}, \varphi_{2}, \ldots, \varphi_{n}\right): X \rightarrow X$ be a surjective mapping. Assume that there is a weighted vector $Q=\left(Q_{1}, Q_{2}, \ldots, Q_{n}\right)$ with every $Q_{i} \in \mathbb{R}_{+}^{k_{i}} \backslash\{0\}$ such that the following conditions are satisfied:

(i) for every $y \in X$, the set $\left\{x \in X: \sum_{i=1}^{n} Q_{i} \cdot\left[V^{i}\left(y_{\hat{i}}, y_{i}\right)-V^{i}\left(y_{\hat{i}}, \varphi_{i}\left(x_{i}\right)\right)\right]>0\right\}$ is convex;

(ii) for every $x \in X$, the function $y \mapsto \sum_{i=1}^{n} Q_{i} \cdot\left[V^{i}\left(y_{\hat{i}}, y_{i}\right)-V^{i}\left(y_{\hat{i}}, \varphi_{i}\left(x_{i}\right)\right)\right]$ is lower semicontinuous on $X$;

(iii) for every $y \in X, \sum_{i=1}^{n} Q_{i} \cdot\left[V^{i}\left(y_{\hat{i}}, y_{i}\right)-V^{i}\left(y_{\hat{i}}, \varphi_{i}\left(y_{i}\right)\right)\right] \leq 0$;

(iv) one of the following conditions holds:

(iv) ${ }_{1}$ for every $N \in\langle X\rangle$, there exists a nonempty compact convex subset $X_{N}$ of $X$ containing $N$ such that

$$
X_{N} \backslash K \subseteq \bigcup_{x \in X_{N}}\left\{y \in X: \sum_{i=1}^{n} Q_{i} \cdot\left[V^{i}\left(y_{\hat{i}}, y_{i}\right)-V^{i}\left(y_{\hat{i}}, \varphi_{i}\left(x_{i}\right)\right)\right]>0\right\} ;
$$

(iv) $)_{2}$ there exists a point $x_{0}=\left(x_{0 i}\right)_{i \in I} \in X$ such that

$$
X \backslash\left\{y \in X: \sum_{i=1}^{n} Q_{i} \cdot\left[V^{i}\left(y_{\hat{i}}, y_{i}\right)-V^{i}\left(y_{\hat{i}}, \varphi_{i}\left(x_{0 i}\right)\right)\right]>0\right\} \subseteq K .
$$

Then $\Gamma$ has at least one weight Nash $\varphi$-equilibrium in $K$ with respect to the weight vector $Q$.

Proof Following the method by Nikaido and Isoda [40], we define the function $S: X \times$ $X \rightarrow \mathbb{R}$ by

$$
S(x, y)=\sum_{i=1}^{n} Q_{i} \cdot\left[V^{i}\left(y_{\hat{i}}, y_{i}\right)-V^{i}\left(y_{\hat{i}}, \varphi_{i}\left(x_{i}\right)\right)\right], \quad(x, y) \in X \times X .
$$

By (i), for every $y \in X$, the set $\{y \in X: S(x, y)>0\}$ is convex. By (ii), for every $x \in X$, the function $y \mapsto S(x, y)$ is lower semicontinuous on $X$. By (iii), for every $y \in X$, we have $S(y, y) \leq 0$. Suppose that (iv) $)_{1}$ holds. Then by (iv) $)_{1}$ and by the definition of $S$, we know that, for every $N \in\langle X\rangle$, there exists a nonempty compact convex subset $X_{N}$ of $X$ containing $N$ such that

$$
X_{N} \backslash K \subseteq \bigcup_{x \in X_{N}}\{y \in X: S(x, y)>0\} .
$$

If (iv) $)_{2}$ is satisfied, then it follows from (iv) $)_{2}$ and from the definition of $S$ that there exists a point $x_{0} \in X$ such that

$$
X \backslash\left\{y \in X: S\left(x_{0}, y\right)>0\right\} \subseteq K .
$$

Thus, by Remark 4.1, all the requirements of Theorem 4.1 with $f=g$ are satisfied. Hence, by Remark 4.1 and by Theorem 4.1 with $f=g$, there exists $\hat{y} \in K$ such that $S(x, \hat{y}) \leq 0$ for 
every $x \in X$; that is,

$$
\sum_{i=1}^{n} Q_{i} \cdot V^{i}\left(\hat{y}_{\hat{i}}, \hat{y}_{i}\right) \leq \sum_{i=1}^{n} Q_{i} \cdot V^{i}\left(\hat{y}_{\hat{i}}, \varphi_{i}\left(x_{i}\right)\right) \quad \text { for every } x \in X .
$$

For every given $i \in I$ and $x_{i} \in X_{i}$, let $x=\left(\hat{y}_{\hat{i}}, x_{i}\right) \in X$. Then we have

$$
\begin{aligned}
Q_{i} \cdot V^{i}\left(\hat{y}_{\hat{i}}, \hat{y}_{i}\right)-Q_{i} \cdot V^{i}\left(\hat{y}_{\hat{i}}, \varphi_{i}\left(x_{i}\right)\right)= & \sum_{j=1}^{n} Q_{j} \cdot\left[V^{j}\left(\hat{y}_{\hat{j}}, \hat{y}_{j}\right)-V^{j}\left(\hat{y}_{\hat{j}}, \varphi_{j}\left(x_{j}\right)\right)\right] \\
& -\sum_{j \neq i} Q_{j} \cdot\left[V^{j}\left(\hat{y}_{\hat{j}}, \hat{y}_{j}\right)-V^{j}\left(\hat{y}_{\hat{j}}, \varphi_{j}\left(x_{j}\right)\right)\right] \\
= & \sum_{j=1}^{n} Q_{j} \cdot\left[V^{j}\left(\hat{y}_{\hat{j}}, \hat{y}_{j}\right)-V^{j}\left(\hat{y}_{\hat{j}}, \varphi_{j}\left(x_{j}\right)\right)\right] \\
\leq & 0 .
\end{aligned}
$$

Therefore, $Q_{i} \cdot R^{i}\left(\hat{y}_{i}, \hat{y}_{i}\right) \leq Q_{i} \cdot V^{i}\left(\hat{y}_{\hat{i}}, \varphi_{i}\left(x_{i}\right)\right)$ for every $i \in I$ and every $x_{i} \in X_{i}$; that is, $\hat{y} \in K$ is a weighted Nash $\varphi$-equilibrium of the game $\Gamma$ with respect to $Q$. This completes the proof.

Remark 5.3 Theorem 5.1 is a new result, which is different from Theorem 3.1 of Wang [37], Theorem 1 of Yuan and Tarafdar [38], Theorem 3 of Yu and Yuan [39], and Theorem 1 of Borm et al. [41]. The main difference is that the underlying strategy spaces in Theorem 5.1 are $\mathrm{CAT}(0)$ spaces which do not possess any linear structure. In addition, on the basis of an existence theorem for weighted Nash equilibrium for multiobjective noncooperative games in the setting of compact finite dimensional spaces, $\mathrm{Lu}$ [42] analyzed the phenomena for the water resources utilizing conflicts among the water users in the lower reaches of Tarim River Basin and revealed the underlying causes of water shortage and water quality deterioration of the lower reaches of Tarim River Basin. We point out that the underlying strategy spaces of multiobjective noncooperative game models in [42] are compact finite dimensional spaces and the payoff functions of players are continuous, which restrict the applicable area of models. In fact, in real world, the situation that the underlying strategy spaces of players are noncompact and nonlinear spaces and the payoff functions of players are discontinuous is very common. So, the multiobjective noncooperative game models in [42] cannot be used to analyze many conflict problems under the situation mentioned above. In contrast with the multiobjective noncooperative game models in [42], the multiobjective noncooperative game model in Theorem 5.1 has two advantages; that is, the strategy spaces of players do not possess any linear and compact structure and the payoff functions of players need not to be continuous. Therefore, by using Theorem 5.1, we can deal with a lot of conflict problems existing in resource utilizing and management under much more mild conditions.

Remark 5.4 (ii) of Theorem 5.1 can be replaced by the following conditions:

(ii)' for every $x \in X$, the function $y \rightarrow \sum_{i=1}^{n} Q_{i} \cdot V^{i}\left(y_{\hat{i}}, \varphi_{i}\left(x_{i}\right)\right)$ is upper semicontinuous on $X$; (ii)" the function $(x, y) \rightarrow \sum_{i=1}^{n} Q_{i} \cdot V^{i}\left(x_{\hat{i}}, y_{i}\right)$ is jointly lower semicontinuous on $X \times X$. 
If $k_{i}=1$ for every $i \in I$, then, by Theorem 5.1, we have the following existence result of Nash $\varphi$-equilibrium for noncooperative games.

Corollary 5.1 Let $\Gamma=\left(X_{i}, f^{i}\right)_{i \in I}$ be a noncooperative game with every $f^{i}$ being the payoff function of player $i$. For every $i \in I$, let $X_{i}$ be a nonempty subset of a $\mathrm{CAT}(0)$ space $\left(E_{i}, d_{i}\right)$ such that $X=\prod_{i \in I} X_{i}$ is a complete $\mathrm{CAT}(0)$ space with the convex hull finite property. Let $K$ be a nonempty compact subset of $X$ and $\varphi=\left(\varphi_{1}, \varphi_{2}, \ldots, \varphi_{n}\right): X \rightarrow X$ be a surjective mapping. Assume that

(i) for every $y \in X$, the set $\left\{x \in X: \sum_{i=1}^{n}\left[f^{i}\left(y_{\hat{i}}, y_{i}\right)-f^{i}\left(y_{\hat{i}}, \varphi_{i}\left(x_{i}\right)\right)\right]>0\right\}$ is convex;

(ii) for every $x \in X$, the function $y \mapsto \sum_{i=1}^{n}\left[f^{i}\left(y_{\hat{i}}, y_{i}\right)-f^{i}\left(y_{\hat{i}}, \varphi_{i}\left(x_{i}\right)\right)\right]$ is lower semicontinuous on $X$;

(iii) for every $y \in X, \sum_{i=1}^{n}\left[f^{i}\left(y_{\hat{i}}, y_{i}\right)-f^{i}\left(y_{\hat{i}}, \varphi_{i}\left(y_{i}\right)\right)\right] \leq 0$;

(iv) one of the following conditions holds:

(iv) $)_{1}$ for every $N \in\langle X\rangle$, there exists a nonempty compact convex subset $X_{N}$ of $X$ containing $N$ such that

$$
X_{N} \backslash K \subseteq \bigcup_{x \in X_{N}}\left\{y \in X: \sum_{i=1}^{n}\left[f^{i}\left(y_{\hat{i}}, y_{i}\right)-f^{i}\left(y_{\hat{i}}, \varphi_{i}\left(x_{i}\right)\right)\right]>0\right\} ;
$$

(iv) $)_{2}$ there exists a point $x_{0}=\left(x_{0 i}\right)_{i \in I} \in X$ such that

$$
X \backslash\left\{y \in X: \sum_{i=1}^{n}\left[f^{i}\left(y_{\hat{i}}, y_{i}\right)-f^{i}\left(y_{\hat{i}}, \varphi_{i}\left(x_{i}\right)\right)\right]>0\right\} \subseteq K .
$$

Then $\Gamma$ has a Nash $\varphi$-equilibrium in $K$.

Remark 5.5 It is interesting to compare Corollary 5.1 with Theorem 4 of Niculescu and Rovența [29] in the following aspects: (1) every $X_{i}$ in Corollary 5.1 is a nonempty subset of a $\operatorname{CAT}(0)$ space $\left(E_{i}, d_{i}\right)$ and it does not need to be compact, where all $\left(E_{i}, d_{i}\right)$ are possibly different; (2) every function $f^{i}$ in Corollary 5.1 does not need to be lower semicontinuous and quasi-convex; (3) the mapping $\varphi$ in Corollary 5.1 does not need to be continuous and affine.

By Theorem 5.1, we can derive an existence theorem of Pareto $\varphi$-equilibrium for multiobjective noncooperative games. In order to do so, we need the following lemma. The proof of this lemma is similar to that of Lemma 2.1 of Wang [37]. For the sake of completeness, we give the proof.

Lemma 5.1 Every normalized weighted Nash $\varphi$-equilibrium $\hat{x} \in X$ with a weight $Q=$ $\left(Q_{1}, Q_{2}, \ldots, Q_{n}\right), Q_{i} \in \mathbb{R}_{+}^{k_{i}} \backslash\{0\}$ (respectively, $Q_{i} \in \operatorname{int}_{\mathbb{R}^{k_{i}}} \mathbb{R}_{+}^{k_{i}}$ ) and $\sum_{j=1}^{k_{i}} Q_{i, j}=1$ for every $i \in I$, is a weak Pareto $\varphi$-equilibrium (respectively, a Pareto $\varphi$-equilibrium) of the game $\Gamma=\left(X_{i}, V^{i}\right)_{i \in I}$.

Proof Let $\hat{x} \in X$ be a normalized weight Nash $\varphi$-equilibrium of the game $\Gamma=\left(X_{i}, V^{i}\right)_{i \in I}$ with a weight $Q=\left(Q_{1}, Q_{2}, \ldots, Q_{n}\right), Q_{i} \in \mathbb{R}_{+}^{k_{i}} \backslash\{0\}$ and $\sum_{j=1}^{k_{i}} Q_{i, j}=1$ for every $i \in I$. We can prove that $\hat{x}$ is a weak Pareto $\varphi$-equilibrium. In fact, suppose the contrary. Then it follows 
from Definition 5.2 that there exist $i_{0} \in I$ and $x_{i_{0}} \in X_{i}$ such that

$$
V^{i}\left(\hat{x}_{\hat{i}_{0}}, \hat{x}_{i_{0}}\right)-V^{i}\left(\hat{x}_{\hat{i}_{0}}, \varphi\left(x_{i_{0}}\right)\right) \in \text { int }_{\mathbb{R}^{k_{i}}} \mathbb{R}_{+}^{k_{i_{0}}}
$$

Since $Q_{i_{0}} \in \mathbb{R}_{+}^{k_{i_{0}}} \backslash\{0\}$, it follows that $Q_{i_{0}} \cdot\left[V^{i}\left(\hat{x}_{\hat{i}_{0}}, \hat{x}_{i_{0}}\right)-V^{i}\left(\hat{x}_{\hat{i}_{0}}, \varphi\left(x_{i_{0}}\right)\right)\right]>0$, which contradicts the assumption that $\hat{x}$ is a normalized weighted Nash $\varphi$-equilibrium with the weight $Q=\left(Q_{1}, Q_{2}, \ldots, Q_{n}\right)$. Hence, $\hat{x}$ is a weak Pareto $\varphi$-equilibrium. Now let $\hat{x}$ be a normalized weight Nash $\varphi$-equilibrium of the game $\Gamma=\left(X_{i}, V^{i}\right)_{i \in I}$ with a weight $Q=$ $\left(Q_{1}, Q_{2}, \ldots, Q_{n}\right), Q_{i} \in$ int $_{\mathbb{R}^{k_{i}}} \mathbb{R}_{+}^{k_{i}}$ and $\sum_{j=1}^{k_{i}} Q_{i, j}=1$ for every $i \in I$. We can show that $\hat{x}$ is a Pareto $\varphi$-equilibrium. In fact, if it were not the case, then by Definition 5.2 , we know that there exist $i_{0} \in I$ and $x_{i_{0}} \in X_{i}$ such that

$$
V^{i}\left(\hat{x}_{\hat{i}_{0}}, \hat{x}_{i_{0}}\right)-V^{i}\left(\hat{x}_{\hat{i}_{0}}, \varphi\left(x_{i_{0}}\right)\right) \in \mathbb{R}_{+}^{k_{i_{0}}} \backslash\{0\}
$$

Since $Q_{i_{0}} \in \operatorname{int}_{\mathbb{R}^{k_{i}}} \mathbb{R}_{+}^{k_{i}}$, it follows that $Q_{i_{0}} \cdot\left[V^{i}\left(\hat{x}_{\hat{i}_{0}}, \hat{x}_{i_{0}}\right)-V^{i}\left(\hat{x}_{\hat{i}_{0}}, \varphi\left(x_{i_{0}}\right)\right)\right]>0$, which contradicts the assumption that $\hat{x}$ is a normalized weighted Nash $\varphi$-equilibrium with the weight $Q=\left(Q_{1}, Q_{2}, \ldots, Q_{n}\right)$. Hence, $\hat{x}$ is a Pareto $\varphi$-equilibrium. This completes the proof.

Remark 5.6 The conclusion of Lemma 5.1 is still true if $\hat{x} \in X$ is a weighted Nash $\varphi$-equilibrium with a weight $Q=\left(Q_{1}, Q_{2}, \ldots, Q_{n}\right)$ satisfying $Q_{i} \in \mathbb{R}_{+}^{k_{i}} \backslash\{0\}$ (respectively, $Q_{i} \in$ int $_{\mathbb{R}^{k_{i}}} \mathbb{R}_{+}^{k_{i}}$ ) for every $i \in I$. We point out that a Pareto $\varphi$-equilibrium is not necessarily a weighted Nash $\varphi$-equilibrium.

Theorem 5.2 Let $\Gamma=\left(X_{i}, V^{i}\right)_{i \in I}$ be a multiobjective game with $V^{i}=\left(f_{1}^{i}, f_{2}^{i}, \ldots, f_{k_{i}}^{i}\right)$. For every $i \in I$, let $X_{i}$ be a nonempty subset of a CAT(0) space $\left(E_{i}, d_{i}\right)$ such that $X=\prod_{i \in I} X_{i}$ is a complete $\mathrm{CAT}(0)$ space with the convex hull finite property. Let $K$ be a nonempty compact subset of $X$ and $\varphi=\left(\varphi_{1}, \varphi_{2}, \ldots, \varphi_{n}\right): X \rightarrow X$ be a surjective mapping. Assume that there is a weighted vector $Q=\left(Q_{1}, Q_{2}, \ldots, Q_{n}\right)$ with every $Q_{i} \in \mathbb{R}_{+}^{k_{i}} \backslash\{0\}$ such that the following conditions are satisfied:

(i) for every $y \in X$, the $\operatorname{set}\left\{x \in X: \sum_{i=1}^{n} Q_{i} \cdot\left[V^{i}\left(y_{\hat{i}}, y_{i}\right)-V^{i}\left(y_{\hat{i}}, \varphi_{i}\left(x_{i}\right)\right)\right]>0\right\}$ is convex;

(ii) for every $x \in X$, the function $y \mapsto \sum_{i=1}^{n} Q_{i} \cdot\left[V^{i}\left(y_{\hat{i}}, y_{i}\right)-V^{i}\left(y_{\hat{i}}, \varphi_{i}\left(x_{i}\right)\right)\right]$ is lower semicontinuous on $X$;

(iii) for every $y \in X, \sum_{i=1}^{n} Q_{i} \cdot\left[V^{i}\left(y_{\hat{i}}, y_{i}\right)-V^{i}\left(y_{\hat{i}}, \varphi_{i}\left(y_{i}\right)\right)\right] \leq 0$;

(iv) one of the following conditions holds:

(iv) 1 for every $N \in\langle X\rangle$, there exists a nonempty compact convex subset $X_{N}$ of $X$ containing $N$ such that

$$
X_{N} \backslash K \subseteq \bigcup_{x \in X_{N}}\left\{y \in X: \sum_{i=1}^{n} Q_{i} \cdot\left[V^{i}\left(y_{\hat{i}}, y_{i}\right)-V^{i}\left(y_{\hat{i}}, \varphi_{i}\left(x_{i}\right)\right)\right]>0\right\}
$$

(iv) 2 there exists a point $x_{0}=\left(x_{0 i}\right)_{i \in I} \in X$ such that

$$
X \backslash\left\{y \in X: \sum_{i=1}^{n} Q_{i} \cdot\left[V^{i}\left(y_{\hat{i}}, y_{i}\right)-V^{i}\left(y_{\hat{i}}, \varphi_{i}\left(x_{0 i}\right)\right)\right]>0\right\} \subseteq K .
$$


Then $\Gamma$ has at least one weak Pareto $\varphi$-equilibrium in $K$. In addition, if $Q=\left(Q_{1}, Q_{2}\right.$, $\left.\ldots, Q_{n}\right)$ with each $Q_{i} \in \operatorname{int}_{\mathbb{R}^{k_{i}}} \mathbb{R}_{+}^{k_{i}}$, then $\Gamma$ has at least one Pareto $\varphi$-equilibrium in $K$.

Proof It follows from Theorem 5.1 that $\Gamma$ has at least a weighted Nash $\varphi$-equilibrium point $\hat{y} \in K$ with respect to the weighted vector $Q$. By Lemma 5.1 and by Remark 5.6, we know that $\hat{y}$ is also a weak Pareto $\varphi$-equilibrium point of $\Gamma$, and $\hat{y}$ is a Pareto $\varphi$-equilibrium point if $Q_{i} \in \operatorname{int}_{\mathbb{R}^{k_{i}}} \mathbb{R}_{+}^{k_{i}}$ for every $i \in I$. This completes the proof.

\section{Competing interests}

The authors declare that they have no competing interests.

\section{Authors' contributions}

All authors contributed equally to the writing of this paper. All authors read and approved the final manuscript.

\section{Author details}

'School of Business, Jiangsu University of Technology, Changzhou, Jiangsu 213001, China. ${ }^{2}$ School of Digital Media, Jiangnan University, Wuxi, Jiangsu, 214122, China. ${ }^{3}$ Department of Mathematical Sciences, The University of Texas at Dallas, Richardson, TX 75080, USA. ${ }^{4}$ Department of Mathematics, Tongji University, Shanghai, 200092, China.

\section{Acknowledgements}

This work was supported by the Planning Foundation for Humanities and Social Sciences of Ministry of Education of China 'Research on utilizing conflict of water resources and initial water right allocation in a river basin based on game theory' (No. 12YJAZH084), the Young \& Middle-Aged Academic Leaders Program of the 'Qinglan Project' of Jiangsu Province, and by Jiangsu Overseas Research \& Training Program for University Prominent Young \& Middle-Aged Teachers and Presidents. The corresponding author (the second author) was also supported by the "Six Talent Peaks Project" of Jiangsu Province (No. DZXX-028). The authors would like to thank the referees for their many valuable suggestions and comments which improved the exposition of this paper.

Received: 20 March 2014 Accepted: 30 July 2014 Published: 21 Aug 2014

\section{References}

1. Browder, FE: The fixed point theory of multi-valued mappings in topological vector spaces. Math. Ann. 177, 283-301 (1968)

2. Fan, K: A generalization of Tychonoff's fixed point theorem. Math. Ann. 142, 305-310 (1961)

3. Yannelis, NC: Maximal elements over non-compact subsets of linear topological spaces. Econ. Lett. 17, 133-136 (1985)

4. Mehta, G: Maximal elements for non-transitive binary relations. Econ. Lett. 14, 163-165 (1984)

5. Yuan, GX-Z: KKM Theory and Applications in Nonlinear Analysis. Dekker, New York (1999)

6. Yuan, GX-Z: The study of equilibria for abstract economics in topological vector spaces - a unified approach. Nonlinear Anal. 37, 409-430 (1999)

7. Lin, LJ, Cheng, SF: Nash-type equilibrium theorems and competitive Nash-type equilibrium theorems. Comput. Math. Appl. 44, 1360-1378 (2002)

8. Luo, Q: Ky Fan's section theorem and its applications in topological ordered spaces. Appl. Math. Lett. 17, 1113-1119 (2004)

9. Lin, L: Variational relation problems and equivalent forms of generalized Fan-Browder fixed point theorem with applications to Stampacchia equilibrium problems. J. Glob. Optim. 53, 215-229 (2012)

10. Kum, S, Wong, MM: An extension of a generalized equilibrium problem. Taiwan. J. Math. 15, 1667-1675 (2011)

11. Raj, VS, Somasundaram, S: KKM-type theorems for best proximity points. Appl. Math. Lett. 25, 496-499 (2012)

12. Park, S: Evolution of the 1984 KKM theorem of Ky Fan. Fixed Point Theory Appl. 2012, 146 (2012)

13. Park, S: A genesis of general KKM theorems for abstract convex spaces: revised. J. Nonlinear Anal. Optim. 4(1), 127-132 (2013)

14. Kirk, WA: Some recent results in metric fixed point theory. J. Fixed Point Theory Appl. 2007(2), 195-207 (2007)

15. Kirk, WA: Remarks on approximates fixed points. Nonlinear Anal. 75, 4632-4636 (2012)

16. Dhompongsa, S, Kaewkhao, A, Panyanak, B: Lim's theorems for multivalued mappings in CAT(0) spaces. J. Math. Anal. Appl. 312, 478-487 (2005)

17. Shahzad, N: Fixed point results for multimaps in CAT(0) spaces. Topol. Appl. 156, 997-1001 (2009)

18. Shabanian, S, Vaezpour, SM: A minimax inequality and its applications to fixed point theorems in CAT(0) spaces. Fixed Point Theory Appl. 2011, 61 (2011). doi:10.1186/1687-1812-2011-61

19. Asadi, M: Fixed points and common fixed points of mappings on CAT(0) spaces. Fixed Point Theory 14, $29-38$ (2013)

20. Kirk, WA: Geodesic geometry and fixed point theory. In: Seminar of Mathematical Analysis (Malaga/Seville, 2002/2003). Colecc. Abierta, vol. 64, pp. 195-225. Univ. Seville Secr. Publ., Seville (2003)

21. Shahzad, N, Markin, J: Invariant approximations for commuting mappings in CAT(0) and hyperconvex spaces. J. Math. Anal. Appl. 337, 1457-1464 (2008)

22. Kirk, WA: Geodesic geometry and fixed point theory II. In: International Conference on Fixed Point Theory and Applications, pp. 113-142. Yokohama Publ., Yokohama (2004)

23. Bridson, MR, Haefliger, A: Metric Spaces of Non-Positive Curvature. Springer, Berlin (1999) 
24. Ballmann, W, Brin, M: Orbihedra of nonpositive curvature. Publ. Math. IHES 82, 170-209 (1995)

25. Dhompongsa, S, Panyanak, B: On $\Delta$-convergence theorems in CAT(0) spaces. Comput. Math. Appl. 56, 2572-2579 (2008)

26. Burago, D, Burago, Y, Ivanov, S: A Course in Metric Geometry. Graduate Studies in Mathematics, vol. 33. Am. Math. Soc., Providence (2001)

27. Beg, I, Abbas, M: An iterative process for a family of asymptotically quasi-nonexpansive mappings in CAT(0) spaces. Novi Sad J. Math. 41, 149-157 (2011)

28. Beg, l, Abbas, M: Common fixed point and best approximation in CAT(0) spaces. Southeast Asian Bull. Math. 36, 11-16 (2012)

29. Niculescu, CP, Rovența, I: Fan's inequality in geodesic spaces. Appl. Math. Lett. 22, 1529-1533 (2009)

30. Bruhat, F, Tits, J: Groupes réductifs sur un corps local. I. Données radicielles valuées. Publ. Math. Inst. Hautes Études Sci. 41, 5-251 (1972)

31. Niculescu, CP, Rovenţa, I: Schauder fixed point theorem in spaces with global nonpositive curvature. Fixed Point Theory Appl. 2009, Article ID 906727 (2009). doi:10.1155/2009/906727

32. Tan, KK, Yuan, XZ: A minimax inequality with applications to existence of equilibrium points. Bull. Aust. Math. Soc. 47, 483-503 (1993)

33. Şahin, A, Başarir, M: On the strong convergence of a modified S-iteration process for asymptotically quasi-nonexpansive mappings in a CAT(0) space. Fixed Point Theory Appl. 2013, 12 (2013). doi:10.1186/1687-1812-2013-12

34. Yang, Z, Pu, YJ: Existence and stability of solutions for maximal element theorem on Hadamard manifolds with applications. Nonlinear Anal. 75, 516-525 (2012)

35. Prokopovych, P: On equilibrium existence in payoff secure games. Econ. Theory 48, 5-16 (2011)

36. Aubin, JP, Frankowska, H: Set-Valued Analysis. Birkhäuser Boston, Boston (1990)

37. Wang, SY: Existence of a Pareto equilibrium. J. Optim. Theory Appl. 79, 373-384 (1993)

38. Yuan, XZ, Tarafdar, E: Non-compact Pareto equilibria for multiobjective games. J. Math. Anal. Appl. 204, 156-163 (1996)

39. Yu, J, Yuan, GX-Z: The study of Pareto equilibria for multiobjective games by fixed point and Ky Fan minimax inequality methods. Comput. Math. Appl. 35, 17-24 (1998)

40. Nikaido, H, Isoda, K: Note on non-cooperative convex games. Pac. J. Math. 5, 807-815 (1955)

41. Borm, PE, Tijs, SH, van der Aarssen, J: Pareto equilibrium in multiobjective games. Methods Oper. Res. 60, 303-312 (1990)

42. Lu, HS: Research on utilizing conflicts of water resources and initial water right allocation in a river basin based on game theory. PhD thesis, Hohai University (2007)

10.1186/1029-242X-2014-320

Cite this article as: Lu et al.: Fixed point theorems in CAT(0) spaces with applications. Journal of Inequalities and Applications 2014, 2014:320

\section{Submit your manuscript to a SpringerOpen ${ }^{\ominus}$ journal and benefit from:}

- Convenient online submission

Rigorous peer review

- Immediate publication on acceptance

Open access: articles freely available online

- High visibility within the field

- Retaining the copyright to your article 